\title{
Molecular mechanism of the pH-dependent calcium affinity in langerin
}

\author{
Jan-O. Joswig ${ }^{1}$, Jennifer Anders ${ }^{1}$, Hengxi Zhang ${ }^{1234}$, Christoph Rademacher ${ }^{1234}$, Bettina G. Keller ${ }^{1 *}$ \\ ${ }^{1}$ Department of Biology, Chemistry, and Pharmacy, Freie Universität Berlin, \\ Arnimallee 22, 14195 Berlin, Germany \\ ${ }^{2}$ Department of Biomolecular Systems, Max Planck Institute of Colloids and Interfaces, \\ Am Mühlenberg 1, 14424 Potsdam, Germany \\ ${ }^{3}$ Department of Pharmaceutical Chemistry, University of Vienna, Althanstraße 14, 1090 Vienna, Austria \\ ${ }^{4}$ Department of Microbiology and Immunobiology, Max F. Perutz Laboratories, University of Vienna, \\ Campus Vienna Biocenter 5, 1030 Vienna, Austria \\ * Corresponding author: bettina.keller@fu-berlin.de
}

Running title: $\mathrm{pH}-$ Dependent calcium affinity in langerin

Keywords: $\mathrm{pH}$ regulation, allosteric regulation, conformational change, calcium-binding protein, pattern recognition receptor (PRR), molecular dynamics, computer modeling 


\section{Abstract}

The C-type lectin receptor langerin plays a vital role in the mammalian defense against invading pathogens. Its function hinges on the affinity to its co-factor $\mathrm{Ca}^{2+}$ which in turn is regulated by the $\mathrm{pH}$. We studied the structural consequences of protonating the allosteric $\mathrm{pH}$-sensor histidine H294 by molecular dynamics simulations (total simulation time: about $120 \mu \mathrm{s}$ ) and Markov models. We discovered a mechanism in which the signal that the $\mathrm{pH}$ has dropped is transferred to the $\mathrm{Ca}^{2+}$-binding site without transferring the initial proton. Instead, protonation of H294 unlocks a conformation in which a protonated lysine side-chain forms a hydrogen bond with a $\mathrm{Ca}^{2+}$-coordinating aspartic acid. This destabilizes $\mathrm{Ca}^{2+}$ in the binding pocket, which we probed by steered molecular dynamics. After $\mathrm{Ca}^{2+}$-release, the proton is likely transferred to the aspartic acid and stabilized by a dyad with a nearby glutamic acid, triggering a conformational transition and thus preventing $\mathrm{Ca}^{2+}$-rebinding.

\section{Introduction}

When pathogens invade a mammal (or more specifically: a human), Langerhans cells capture some of the pathogens, process them, and present antigens to the adaptive immune system. The swift activation of the adaptive immune system is critical for the survival of the mammal, and langerin plays a vital role in this process. Langerin is a transmembrane carbohydrate receptor, which is expressed by Langerhans cells of mammalian skin and mucosa $(1,2)$. It belongs to the class of type II C-type lectin receptors (3, 4). It detects pathogens such as influenza virus (5), measles virus (6), HIV (7), fungi (8), mycobacteria (9), and bacteria (10).

Langerin recognizes these pathogens by binding to carbohydrates on the pathogen surface. Its carbohydrate binding pocket contains a $\mathrm{Ca}^{2+}$-cation as co-factor that is essential for carbohydrate binding, and thus for the capture of pathogens. After the initial binding event, the pathogen is captured in an endocytic vesicle, and langerin releases the pathogen into the endosome (Fig. 1a) $(1,2,7,11)$. This cargo release is triggered by a drop of $\mathrm{pH}$ from 7 in the extracellular medium to 5.5 to 6 in the early endosome (12) and by a substantial drop in the $\mathrm{Ca}^{2+}$-concentration from about 1 to $2 \mathrm{mM}$ to a value in the micro molar range (13-15).

The $\mathrm{pH}$-dependent cargo-release is accomplished by a fascinating mechanism in which various chemical equilibria are carefully balanced. To be able to release the cargo into the more acidic endosome, the carbohydrate affinity of langerin needs to be $\mathrm{pH}$-dependent. However, the change in $\mathrm{pH}$ does not affect the carbohydrate binding itself. Instead langerin

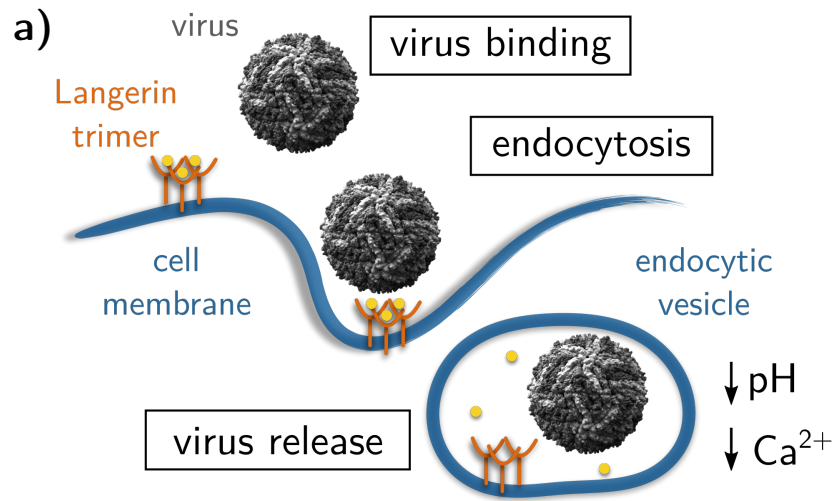

b)

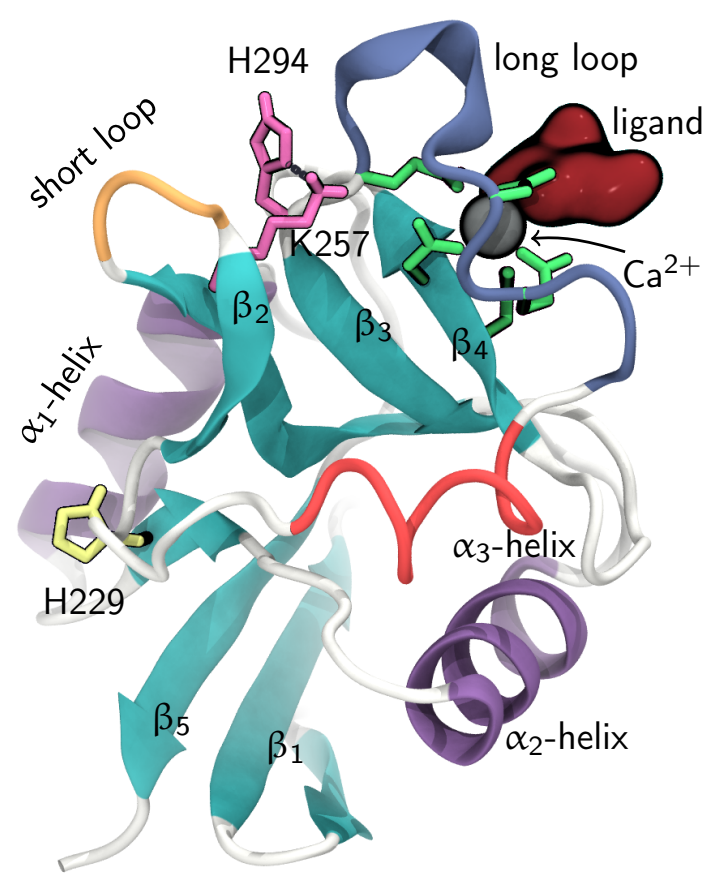

Figure 1: a) Langerin's function as an endocytic pattern recognition receptor (16). b) Langerin carbohydrate recognition domain (PDB-ID 3p5g (17)).

depends on a $\mathrm{Ca}^{2+}$ co-factor for carbohydrate binding, and the observed $\mathrm{pH}$-dependence of the carbohydrate affinity is caused by an underlying $\mathrm{pH}$-dependence of the $\mathrm{Ca}^{2+}$ affinity (18). We previously showed that the $\mathrm{Ca}^{2+}$-affinity is lower at $\mathrm{pH} 6$ than at $\mathrm{pH}$ 7. The $\mathrm{pH}$-sensitivity, measured as the difference in the $\mathrm{Ca}^{2+}$-binding free-energies, is $\Delta \Delta G=5.1 \mathrm{~kJ} \mathrm{~mol}^{-1}$ (18). At high $\mathrm{Ca}^{2+}$-concentrations (10 $\mathrm{mM})$ the carbohydrate affinity ceases to be $\mathrm{pH}$-dependent, because the excess in $\mathrm{Ca}^{2+}$-ions outweighs any change in $\mathrm{Ca}^{2+}$-affinity due to a change in $\mathrm{pH}$. However, in the endosome the $\mathrm{Ca}^{2+}$-concentration is low. Thus, the drop in $\mathrm{pH}$ from the extracellular medium to the endosome causes a decrease in $\mathrm{Ca}^{2+}$-affinity, and the unbinding of the $\mathrm{Ca}^{2+}$ co-factor leads to the dissociation of the carbohydrate ligand and to the release of the pathogen. Similar, $\mathrm{pH}$-sensitivities of either ligand or $\mathrm{Ca}^{2+}$-affinities have been observed for 
several other C-type lectins (19), including ASGPR (14, 20, 21) the macrophage mannose receptor (22), DC-SIGN and DC-SIGNR (23-26), and LSECtin (27) (example structures in SI Fig. 32). In DC-SIGNR and LSECtin, which have a different biological role than langerin, a drop in $\mathrm{pH}$ causes an increase in ligand affinity. The mechanisms underlying the regulation by the $\mathrm{pH}$ in $\mathrm{C}$-type lectins are highly diverse and not yet studied in detail.

The observation that the $\mathrm{Ca}^{2+}$-affinity in C-type lectins is $\mathrm{pH}$-dependent is surprising. First, when a carbohydrate (and attached to it an entire virus) is bound to a C-type lectin, the $\mathrm{Ca}^{2+}$-binding site is almost certainly not solvent exposed. Second, the $\mathrm{Ca}^{2+}$-ion in C-type lectins is coordinated by either aspartate or glutamate side-chains, whose reference $\mathrm{p} K_{\mathrm{a}}$-values (28) (in water at $25^{\circ} \mathrm{C}$ ) are 3.71 (aspartate) and 4.15 (glutamate). By themselves, these residues are not sensitive to a change in $\mathrm{pH}$ from 7 to 6 . Pairs of acidic residues can in principle form a protonated dyad, which is the close arrangement of two residues with acidic sidechains such that protonation of their carboxyl groups is coupled. This results in an increased $\mathrm{p} K_{\mathrm{a}}$ of the protonated residue, stabilized by the unprotonated form of the other group. Prominent examples of this effect are found in the proteins HIV-1 protease $(29,30)$, BACE-1 (31) BACE-2, and CatD, where it can increase the $\mathrm{p} K_{\mathrm{a}}$ of aspartic acid from its reference value to 5.2 (32). The presence of organic ligands can increase these values further (33). However, a protonated dyad can only form if $\mathrm{Ca}^{2+}$ has already left the binding pocket. So the question arises: how do C-type lectins sense a change in $\mathrm{pH}$, and how does this lead to the release of $\mathrm{Ca}^{2+}$ ?

For langerin we previously identified the histidine residue $\mathrm{H} 294$ as a partial $\mathrm{pH}$ sensor that regulates the $\mathrm{Ca}^{2+}$-affinity (18). The reference $\mathrm{p} K_{\mathrm{a}}$ of histidine is 6.04 (in water at $\left.25^{\circ} \mathrm{C}\right)(28)$ which makes it sensitive to a $\mathrm{pH}$ change from 7 to 6 . When $\mathrm{H} 294$ is mutated to A294, the $\mathrm{pH}-$ sensitivity is about $40 \%$ smaller than in the wild-type $\left(\Delta \Delta G=3.1 \mathrm{~kJ} \mathrm{~mol}^{-1}\right.$ upon a change in $\mathrm{pH}$ from 7 to 6 ). Because the histidine side-chain points away from the $\mathrm{Ca}^{2+}$ binding site, it is unlikely that the decrease in $\mathrm{Ca}^{2+}$-affinity is caused by electrostatic repulsion between the protonated histidine and the $\mathrm{Ca}^{2+}$-cation. This mechanism has been suggested for the C-type lectin ASGPR, in which however the histidine $\mathrm{pH}$-sensor is located directly underneath the $\mathrm{Ca}^{2+}$-binding pocket (SI Fig. 32d) (21). Instead we showed - by combining NMR-experiments, site-directed point mutations and molecular dynamics simulations - that $\mathrm{H} 294$ is at the center of an allosteric network that contains the $\mathrm{Ca}^{2+}$-binding site. More specifically, in its unprotonated form $\mathrm{H} 294$ forms a hydrogen bond with lysine K257, which is also present in the known crystal structures of langerin
(17). This hydrogen bond cannot be formed if H294 is protonated, and the allosteric mechanism that regulates the $\mathrm{Ca}^{2+}$-affinity likely hinges on this hydrogen bond.

Yet, protonation of $\mathrm{H} 294$ is only the initial detection that the surrounding medium has changed. Even though we identified the residues that are involved in the allosteric network, we do not yet understand how the protonation of $\mathrm{H} 294$ could ultimately affect the $\mathrm{Ca}^{2+}$-binding pocket. Several allosteric effects have been reported for C-type lectins (see ref. (34) for a recent review), but little is known about their underlying molecular mechanisms that could be applied to the situation in langerin. The goal of this study is to elucidate how the protonation of $\mathrm{H} 294$ changes the conformational ensemble of langerin, and to investigate the effect these conformational changes have on the $\mathrm{Ca}^{2+}$ binding pocket. A model of how the signal, that the $\mathrm{pH}$ has changed, traverses the allosteric network to the buried $\mathrm{Ca}^{2+}$-binding site and triggers the $\mathrm{Ca}^{2+}$-release might serve as a blueprint for understanding how $\mathrm{pH}$-sensitive ligand binding is achieved in C-type lectins and other proteins.

\section{Results and discussion}

\section{Structure of the langerin carbohydrate recognition domain}

Langerin forms a homotrimer. The monomers consist of a short cytoplasmic tail, a transmembrane region and a long alpha-helical neck (residues 56 to 197) extending into the extracellular milieu, which carries the C-terminal carbohydrate recognition domain $(17,19)$. The carbohydrate recognition domain has the typical C-type lectin domain fold (Fig. 1b) (4) which consists of two extended $\beta$-sheets (turquoise), each composed of three single strands. The two $\beta$-sheets are flanked by three $\alpha$-helices (purple, $\alpha_{3}$ in red). The carbohydrate binding pocket which contains the $\mathrm{Ca}^{2+}$-binding site is located on top of the $\beta_{4}$-strand. One residue from this $\beta$-sheet directly binds to the $\mathrm{Ca}^{2+}$-ion: D308. Additionally, the $\mathrm{Ca}^{2+}$-ion is held in place by E293 and E285 in the long-loop (blue), which coordinate to $\mathrm{Ca}^{2+}$ from the side. E285 is part of a conserved EPN-motif (E285, P286, N287 in langerin), which determines the selectivity for mannose, fucose and glucose over galactose $(19,35$, 36). The $\mathrm{pH}$ sensor $\mathrm{H} 294$ (pink) is located at the end of the long-loop. If its side-chain is unprotonated, it forms a hydrogen bond to K257 (also pink) in the short-loop (orange). The allosteric network that regulates the $\mathrm{Ca}^{2+}$ affinity comprises the long- and the short-loop (18). H229 (yellow) is the only other histidine residue in the langerin 

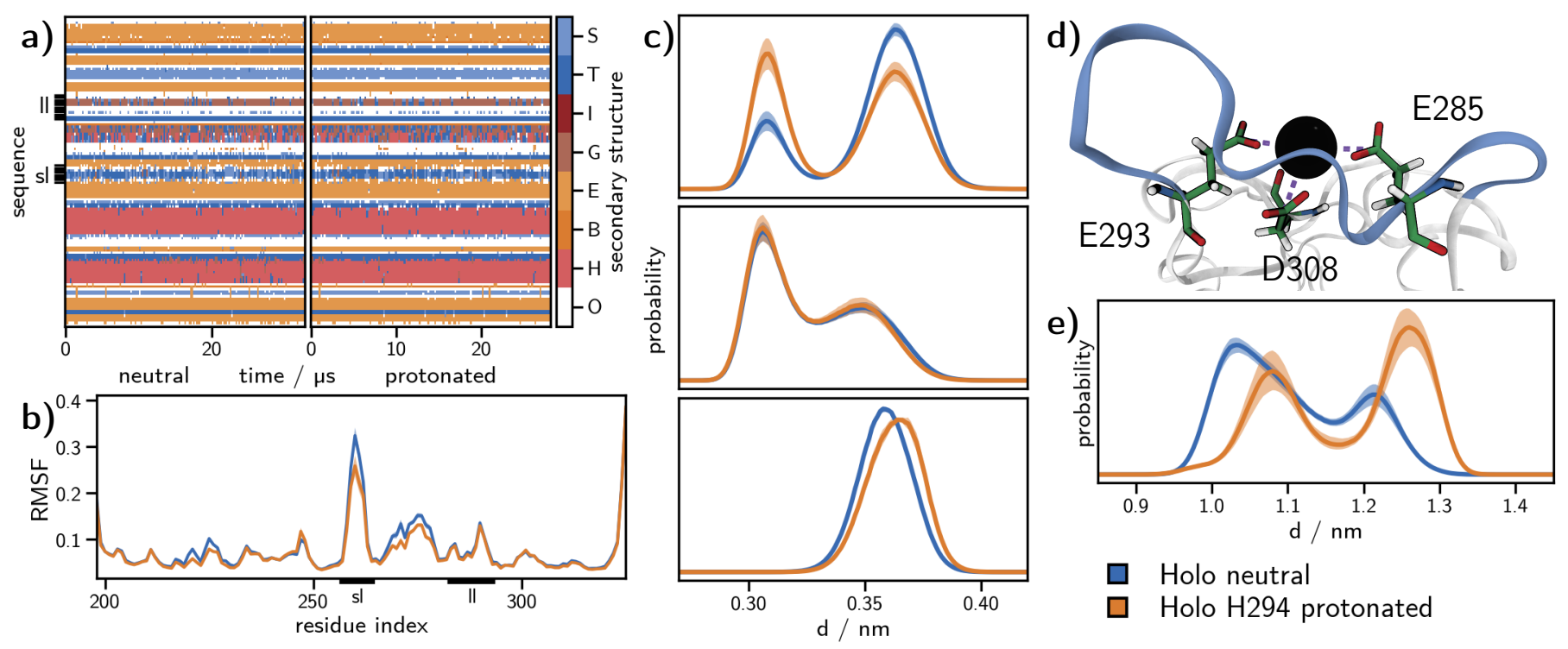

Figure 2: a) Analysis by the hydrogen bond estimation algorithm DSSP of the secondary structure in the neutral (left) and the protonated holo-state (right). Legend: $\mathrm{S}$ - bend, $\mathrm{T}$ - hydrogen bonded turn, I- 5-helix, G - 3-helix, E- extended strand, part of $\beta$-ladder, B: isolated $\beta$-bridge, $\mathrm{H}$ : $\alpha$-helix, O: unassigned. b) $\mathrm{C}_{\alpha}$-root-mean-square fluctuation (RMSF); sl: short-loop, 11: long-loop. c) Carboxyl carbon-Ca ${ }^{2+}$ distance histograms for E285 (upper graph), E293 (middle graph), and D308 (lower graph). d) Structure of the $\mathrm{Ca}^{2+}$-binding site showing the distances plotted in c) with dashed lines. e) Histogram of the minimum distance between $\mathrm{Ca}^{2+}$ and the side-chain $\mathrm{N}$-atoms $\left(\mathrm{N}_{\delta}\right.$ and $\left.\mathrm{N}_{\epsilon}\right)$ of $\mathrm{H} 294$. Solid lines: Mean of the histograms calculated for each simulation replica. Shaded area: $95 \%$ confidence interval of the mean obtained by bootstrapping (1000 samples).

carbohydrate recognition domain. A pathogen would bind via a carbohydrate ligand (dark red) to langerin, and would be separated from the $\mathrm{pH}$ sensor by the long-loop. If $\mathrm{Ca}^{2+}$ is bound to langerin, we will call the system holo-langerin, otherwise apo-langerin.

\section{The effect of $\mathrm{H294}$ protonation on the conforma- tional ensemble}

We conducted $31 \mu$ s of molecular dynamics simulations of holo-langerin, in which all residues were protonated according to their default protonation state at $\mathrm{pH} 7$, i.e. $\mathrm{H} 294$ was unprotonated, and the overall protein was neutral (neutral state). We compare these simulations to $27 \mu$ s of hololangerin, in which $\mathrm{H} 294$ was protonated (protonated state). Protonation of $\mathrm{H} 294$ has no influence on the secondary structure of langerin (Fig. 2a, Fig. 1 in the SI). Thus, any conformational change due to the protonation of $\mathrm{H} 294$ affects the side-chains, or those residues that are not assigned to a specific secondary structure, i.e. the loop regions.

One way a conformational change in the loop regions could manifest itself, is by a change of the loop flexibility. This is however not corroborated by the root-mean-square fluctuations of the individual residues (Fig. 2b). The shortloop (sharp peak around residue 260) and the $\alpha_{3}$-helix (broad peak around residue 275) are more rigid in the protonated state, but the difference is very small. The flexibility in all other regions of the protein, and in particular the long-loop region, does not change upon protonation.

To gauge whether protonation of $\mathrm{H} 294$ has an influence on the conformation of the $\mathrm{Ca}^{2+}$-binding site, we measured the distance distribution between the carboxyl group of the $\mathrm{Ca}^{2+}$-coordinating residues - E285, E293, and D308 - and the $\mathrm{Ca}^{2+}$-ion (Fig. 2c-d). For E293 and D308 the differences are too minor to explain the observed difference in $\mathrm{Ca}^{2+}$-affinity. For E285 the distribution shifts slightly to lower distances and thus to a potentially tightly bound $\mathrm{Ca}^{2+}$-ion, not explaining it either. The distance difference between the two populated states is about $0.05 \mathrm{~nm}$.

Yet, we know from our previous analyses (18) that protonation of H294 causes a significant shift in the conformational ensemble, and this is again confirmed by the distance distributions between the $\mathrm{H} 294$ side-chain and the $\mathrm{Ca}^{2+}$-ion in the neutral and the protonated state (Fig. 2e). In the protonated state the distribution shifts to larger distances, well beyond $1 \mathrm{~nm}$. At this distance, we do not expect a significant influence of the positively charged H294 side-chain on the $\mathrm{Ca}^{2+}$-ion, considering that $\mathrm{H} 294$ is located on the protein surface and that the dielectric constant between the two interacting groups is relatively high (see SI Fig. 20 
a)

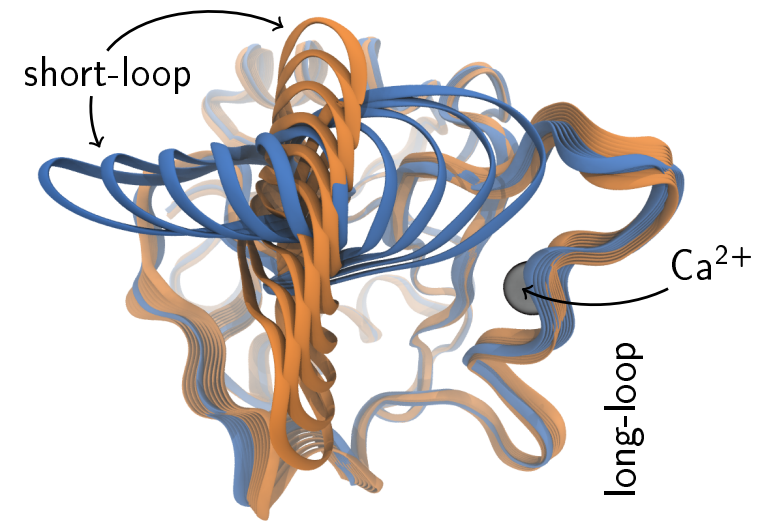

c)

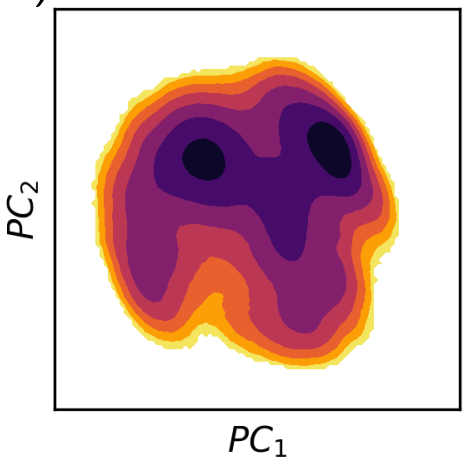

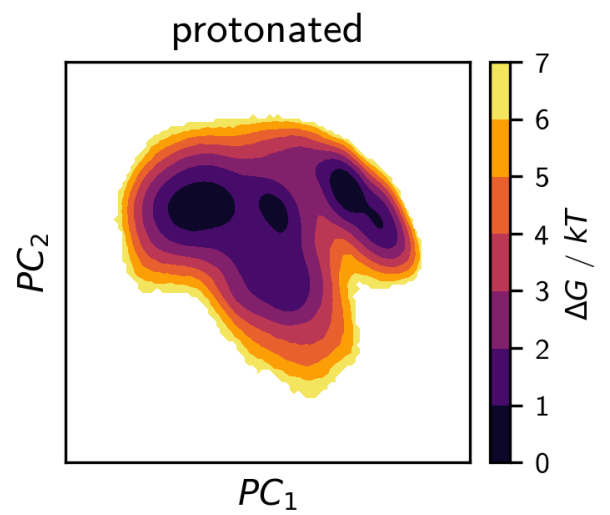
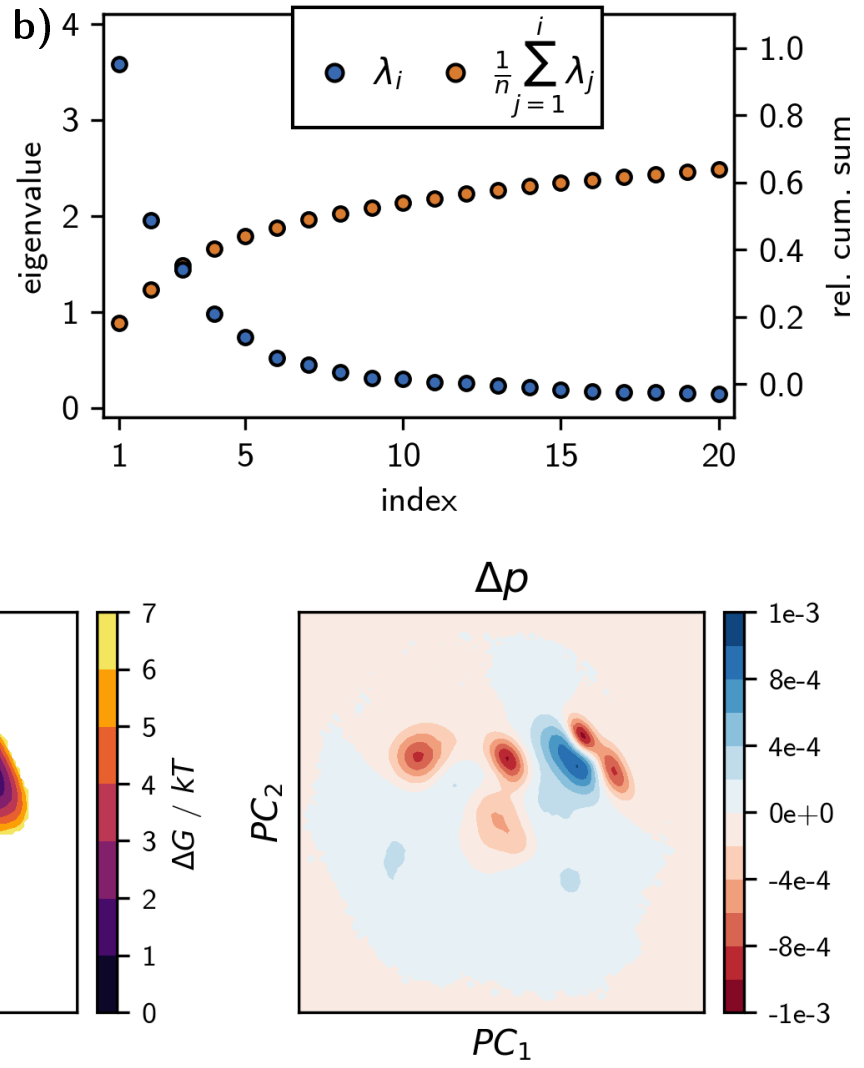

Figure 3: a) Structural interpolations along the first two principle components. b) Eigenvalue spectrum of the principle component analysis (blue dots) and the cumulative sum normalized by the total sum of all $N$ eigenvalues $n=\sum_{i=1}^{N} \lambda_{i}$ (orange dots). c) Free energy surfaces from the 2D projections of the individual holo-langerin trajectories onto principle components 1 and 2 and difference plot of the underlying probability distributions (neutral - protonated).

and 21 for an assessment of the Coulomb interaction) (37). Thus, we can rule out that the decrease in $\mathrm{Ca}^{2+}$-affinity is caused by direct Coulomb repulsion between the protonated $\mathrm{H} 294$ and the $\mathrm{Ca}^{2+}$-cation.

To uncover which residues besides $\mathrm{H} 294$ are involved in the conformational shift, one needs to compare the two conformational ensembles. This cannot be accomplished in the full high-dimensional conformational space. Instead one needs to project the two ensembles into a low-dimensional space that is representative of both systems. Principal component analysis (38) identifies low-dimensional spaces which preserve the directions of the largest conformational variance (39). To be able to directly compare the neutral and the protonated ensemble, we combined the simulations in the two protonation states to obtain a joint principal component space. The principal component with the largest variance represents the opening and closing of the gap between shortloop and long-loop (blue sequence of structures in Fig. 3a). The second principal component represents a sideways shear motion of the short-loop (orange sequence of structures in Fig. 3a). This is in line with our previous finding that the allosteric network is centered on these two loops (18). Even though the two principal components cover only about $28 \%$ of the total structural variance (Fig. 3b), they represent the conformational fluctuations that are most sensitive to a protonation of H294. Separate principal component analyses of the two protonation states yielded principal components that were almost identical, indicating that the joint principal components are a faithful representation of largest variances for both protonation states. Fig. 3c shows the free energy surface of the two systems in the space of the first two joint principal components. The free-energy surface of the unprotonated system is shallow with two minima corresponding to the open and closed state of the short and the long-loop. Upon protonation, the free energy surface becomes much steeper and more structured. One can discern at least three minima. The difference plot of the probability densities in the neutral and protonated state (Fig. $3 \mathrm{c}$ to the right) shows these emerging conformations in red.

We extracted the highly populated regions by clustering in the space of the first two principal components using the density-based common-nearest-neighbors cluster algorithm (40-42), and characterized the hydrogen bond pattern of the 
short- and long-loop residues in each of the clusters (Fig. 4). Figs. $4 \mathrm{c}$ and $4 \mathrm{~d}$ show a subset of the full analysis (see SI Fig 10) focusing on fluctuating hydrogen bonds. In the neutral state, the clusters have essentially the same hydrogen bond populations as the total ensemble, which is consistent with the shallow free-energy surface in Fig. 3c.

The situation is different in the protonated state. Here, each of the four clusters is stabilized by a hydrogen bond pattern that is distinctively different from the hydrogen bond pattern of the total distribution (Fig. 4b). This indicates that upon protonation of H294 several distinct short-loop/longloop conformations emerge.
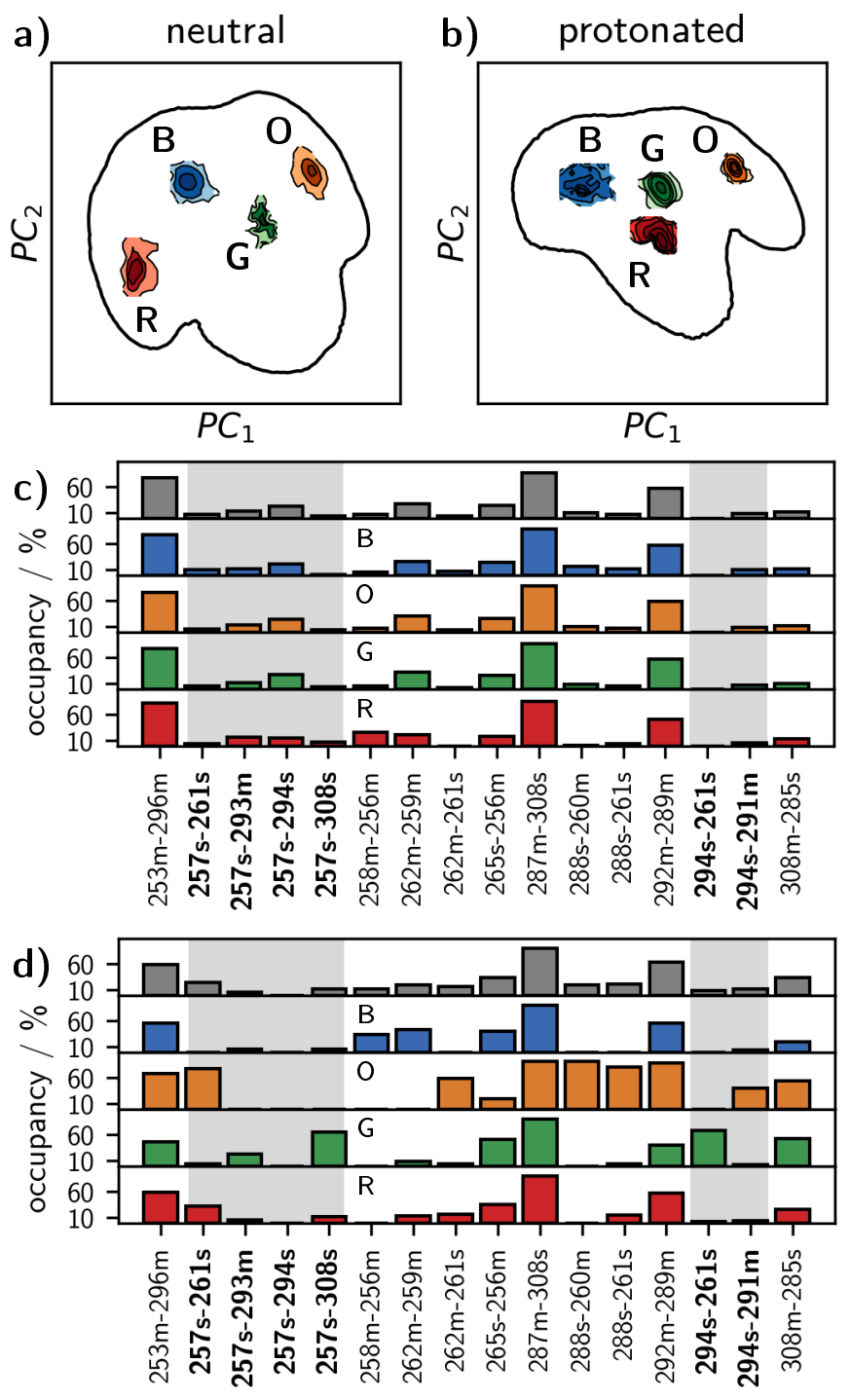

Figure 4: Four most populated clusters in the principalcomponent free energy surface of a) the neutral and b) the protonated holo-langerin. Per cluster hydrogen bond occupancy in c) neutral and d) protonated holo-langerin (populations in the full ensemble in gray). Hydrogen bonds involving K257 or H294 are highlighted by a gray background.
The most striking change arises in the green $(\mathrm{G})$ cluster: the hydrogen bond between the side-chain of K257 and the side-chain of D308, which is barely populated in the unprotonated state (4.2\%), is populated to $65.4 \%$ in this cluster $(12.9 \%$ in the ensemble). In parallel, the side-chain of the now protonated $\mathrm{H} 294$ forms a hydrogen bond with the carboxyl group of E261. The structure is further stabilized by a hydrogen bond between the side-chain of S265 and the main-chain of T256. Note the significance of this finding: the K257 side-chain, which is no longer engaged in a hydrogen-bond with $\mathrm{H} 294$, forms a new hydrogen bond with the $\mathrm{Ca}^{2+}$-coordinating residue D308, and thereby moves a proton into the vicinity of the $\mathrm{Ca}^{2+}$-binding pocket.

The conformation of the orange $(\mathrm{O})$ cluster is complementary to that of the green cluster. The side-chain of K257 forms a hydrogen bond with the carboxyl group of E261, while $\mathrm{H} 294$ engages in a hydrogen bond to the backbone carbonyl oxygen of N291. The conformation is stabilized by hydrogen bonds between the side-chain of N288 and the backbone carbonyl oxygen of M260 and the side-chain of E261. N288 is located in the center of the long-loop, and E261 is located in the center of the short-loop. Thus, these two hydrogen bonds closely connect the two loops explaining why this structure appears in the closed-loop region of the free-energy surface. The main-chain-mainchain hydrogen bond between N292 and A289 additionally stabilizes this structure.

The blue (B) cluster is an open-loop structure in which neither K257 nor H294 are engaged in one of the considered hydrogen bonds. It features the $258 \mathrm{~m}-256 \mathrm{~m}$ and $262 \mathrm{~m}-$ $259 \mathrm{~m}$ hydrogen bonds within the short-loop. The red (R) cluster is a slightly sheared structure in which the K257 side-chain partly forms a hydrogen bond to the carboxyl group of E261 and partly to the carboxyl group of D308.

Three hydrogen bonds in Fig. 4 directly involve $\mathrm{Ca}^{2+}$ coordinating residues. First, we already discussed the hydrogen bond K257-D308. Second, the hydrogen bond between the main-chain of N287 and the side-chain of D308 is important for the stability of the long-loop fold. It is occupied to about $90 \%$ in both protonation states. Third, population of the hydrogen bond between the main-chain amid group of D308 and the carboxyl group of E285 is increased in the protonated state. This is particularly true for cluster $\mathrm{G}$ (green) and $\mathrm{O}$ (orange). This hydrogen bond might compete with the coordination of E285 to $\mathrm{Ca}^{2+}$ and thereby might contribute to the observed decrease in $\mathrm{Ca}^{2+}$ affinity. In both, the neutral and the protonated system, the bonds N288s-M260m, N288s-E261s, K257s-E261s and G262m-E261s are strongly correlated (see SI Fig 10). In the protonated state a strong correlation between K257s-D308s and H294s-E261s arises, indicating that these two hydrogen 
a)

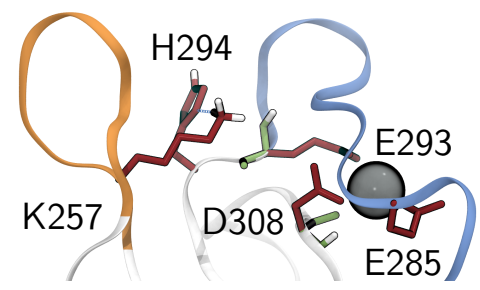

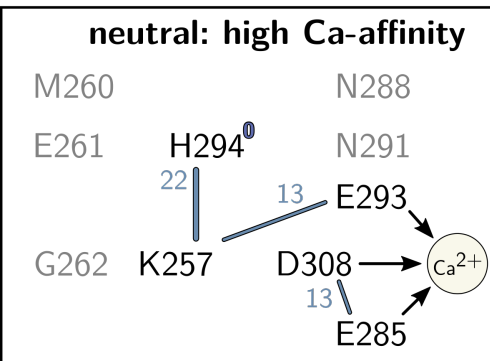

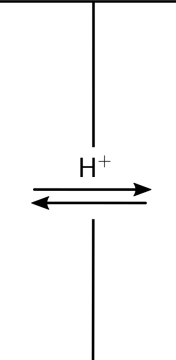

b)

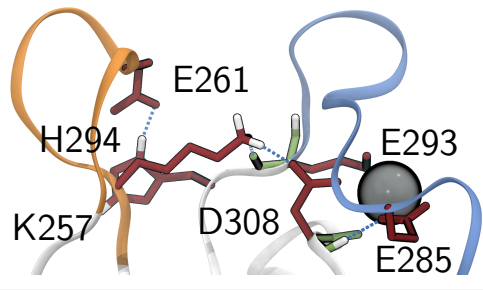

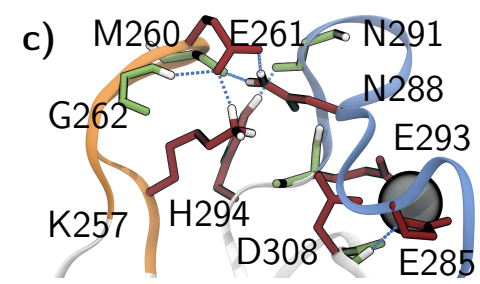
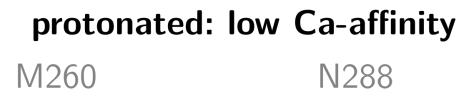

$\mathrm{E} 261 \underset{69}{\mathrm{H}} 294^{+}$

N291

G262

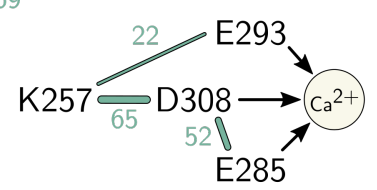

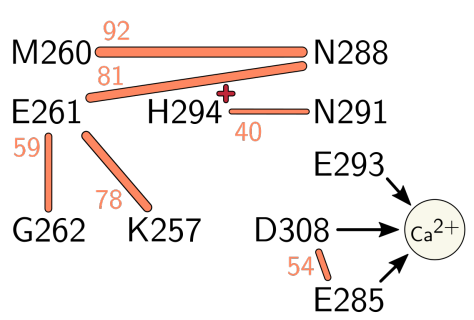

Figure 5: Allosteric mechanism for the $\mathrm{pH}$-sensitive $\mathrm{Ca}^{2+}$-affinity in langerin. a) neutral state, b) cluster $\mathrm{G}$ (green) in the protonated state, and c) cluster $\mathrm{O}$ (orange) in the protonated state. Lines: hydrogen bonds with population in percent. Arrows: Coordination between carboxyl groups and $\mathrm{Ca}^{2+}$.

bonds are formed and broken simultaneously.

\section{A mechanism for the $\mathrm{pH}$-sensitive $\mathrm{Ca}^{2+}$-affinity in langerin}

We are now ready to propose a mechanism that explains how protonation of $\mathrm{H} 294$ can lead to a decrease in $\mathrm{Ca}^{2+}$-affinity. In the neutral state, K257 and H294 form a hydrogen bond which is populated over a wide range of conformations. We also observe a weak hydrogen bond of the K257 side-chain to the main-chain of the $\mathrm{Ca}^{2+}$-coordinating residue E293, but direct hydrogen bonds to the $\mathrm{Ca}^{2+}$-coordinating carboxyl groups are hardly ever formed (Fig. 5a). Upon a drop of $\mathrm{pH}$ from 7 to 6 , the side-chain of $\mathrm{H} 294$ is protonated in accordance with its $\mathrm{p} K_{\mathrm{a}}: \mathrm{H} 294$ is the initial $\mathrm{pH}$ sensor. The protonation of $\mathrm{H} 294$ changes the hydrogen bond pattern between the short and the long-loop. In particular the sidechains of H294 and K257 form new contacts, which gives rise to previously inaccessible conformations. Cluster O (orange) and cluster G (green) exhibit mutually exclusive hydrogen bond patterns. In cluster $\mathrm{O}$, multiple hydrogen bonds connect the short and the long-loop causing a closed loop conformation. The positively charged side-chain of K257 forms a hydrogen bond to the negatively charged side-chain of E261. But similar to the neutral state, there is no direct hydrogen bond to the $\mathrm{Ca}^{2+}$-coordinating carboxyl groups (Fig. 5c). This is different in cluster G. Here the positively charged side-chain of $\mathrm{H} 294$ forms a hydrogen bond with the negatively charged carboxyl group of E261. Simultaneously, the positively charged side-chain of K257 forms a hydrogen bond with the carboxyl group of D308 (Fig. 5b). This hydrogen bond withdraws electron density from the coordinative bond between D308 and $\mathrm{Ca}^{2+}$, and thereby reduces the $\mathrm{Ca}^{2+}$-affinity. It is even conceivable that the proton is transferred entirely to the carboxyl group of D308 (43). We thus propose that cluster G (green) is responsible for the decrease in $\mathrm{Ca}^{2+}$-affinity at $\mathrm{pH} 6$.

In this mechanism, K257 acts as a proton reservoir. The initial detection of a $\mathrm{pH}$ change via protonation of $\mathrm{H} 294$ leads to the cluster $\mathrm{G}$, in which $\mathrm{K} 257$ moves a proton into the vicinity of the $\mathrm{Ca}^{2+}$-binding site and locally increases the proton concentration. Thus, the signal that the $\mathrm{pH}$ has changed is allosterically transferred to the $\mathrm{Ca}^{2+}$-binding pocket without transferring the actual proton that triggered the mechanism.

A crucial assertion in the proposed mechanism is, that the life-time of cluster $\mathrm{G}$ (green) represents a distinct conformation, that is stable enough for the $\mathrm{Ca}^{2+}$-ion to leave the binding pocket. The fact that cluster $\mathrm{G}$ corresponds to a free-energy minimum in the space of the principal components hints at a stable conformation. But because the principal components maximize the spatial variance and not the variance in time, this is not sufficient to be certain.

Fig. 6a shows the distance distribution between the K257 and D308 side-chain for the neutral and the protonated state. In both protonation states, the maximum at short distances around $0.2 \mathrm{~nm}$ is well separated from the maximum at larger distances.

In the neutral state, the short distances are populated only in $4.3 \%$ of all simulated conformations, which increases to $13.2 \%$ when $\mathrm{H} 294$ is protonated. This is in line with the increase of population in the K257-D308 hydrogen bond from $4.2 \%$ to $12.9 \%$. We obtain the same results, when plotting the distance between the K257 side-chain amine 
and the $\mathrm{Ca}^{2+}{ }_{-}$ion in Fig. 6b. Thus, cluster $\mathrm{G}$ (green) indeed represents a distinct conformation.
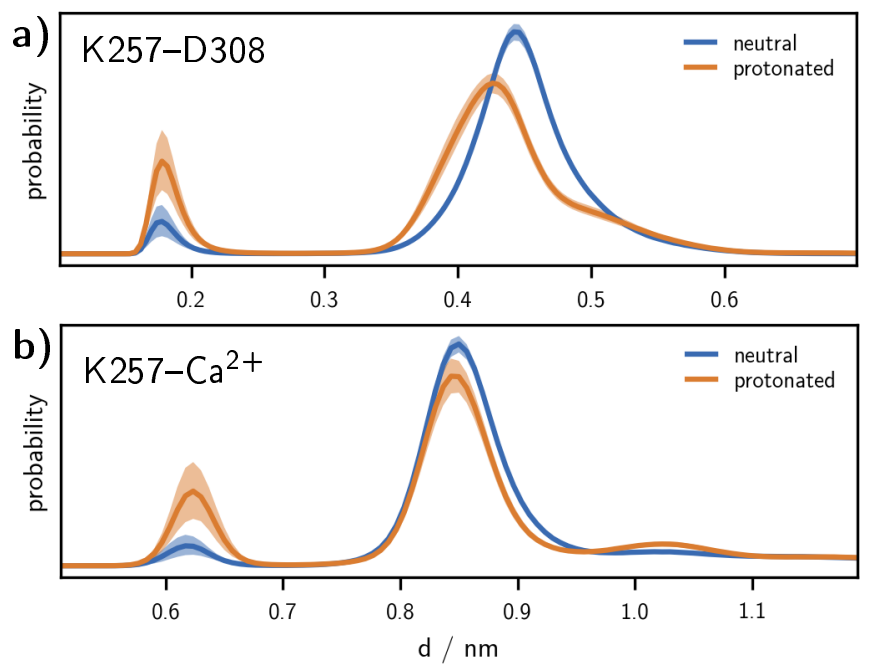

Figure 6: a) K257-D308 side-chain distance distribution. b) K257 side-chain amine $-\mathrm{Ca}^{2+}$ distance distribution. Solid lines: Mean of the histograms calculated for each simulation replica. Shaded area: $95 \%$ confidence interval of the mean obtained by bootstrapping (1000 samples).

To assess the stability of conformations in cluster $G$ (green), and to relate its formation to other dynamic processes in the protein, we constructed a core-set Markov model of the conformational dynamics (44-46). In Markov models, the conformational space is discretized into states and the conformational dynamics are modeled as Markov transitions within a lag time $\tau$ between pairs of these states, where the transition probabilities are obtained from molecular dynamics simulations. From the eigenvectors and eigenvalues of the Markov-model transition matrix one obtains long-lived conformations as well as the hierarchy of the free-energy barriers separating them. The special feature of core-set Markov models is, that the states are confined to the regions close to the minima of the free-energy surface, i.e. so-called core-sets, whereas the regions between these minima are modeled by committor functions. This reduces the discretization error of the model considerably.

The Markov-model construction is preceded by a dimensionality reduction of the conformational space using the time-independent component analysis $(47,48)$. Timeindependent components (tIC) maximize the variance within lag-time $\tau$ rather than the instantaneous variance maximized by principal components. A projection into a lowdimensional tIC-space can thus be interpreted as projection into the space of the slowly varying coordinates of the system. Fig. 7a shows the free-energy surface of the protonated system projected into the space of the first and the second tIC (see SI for other projections), and Fig. 7b shows the projection of cluster $\mathrm{G}$ (green) and $\mathrm{O}$ (orange) into this space.
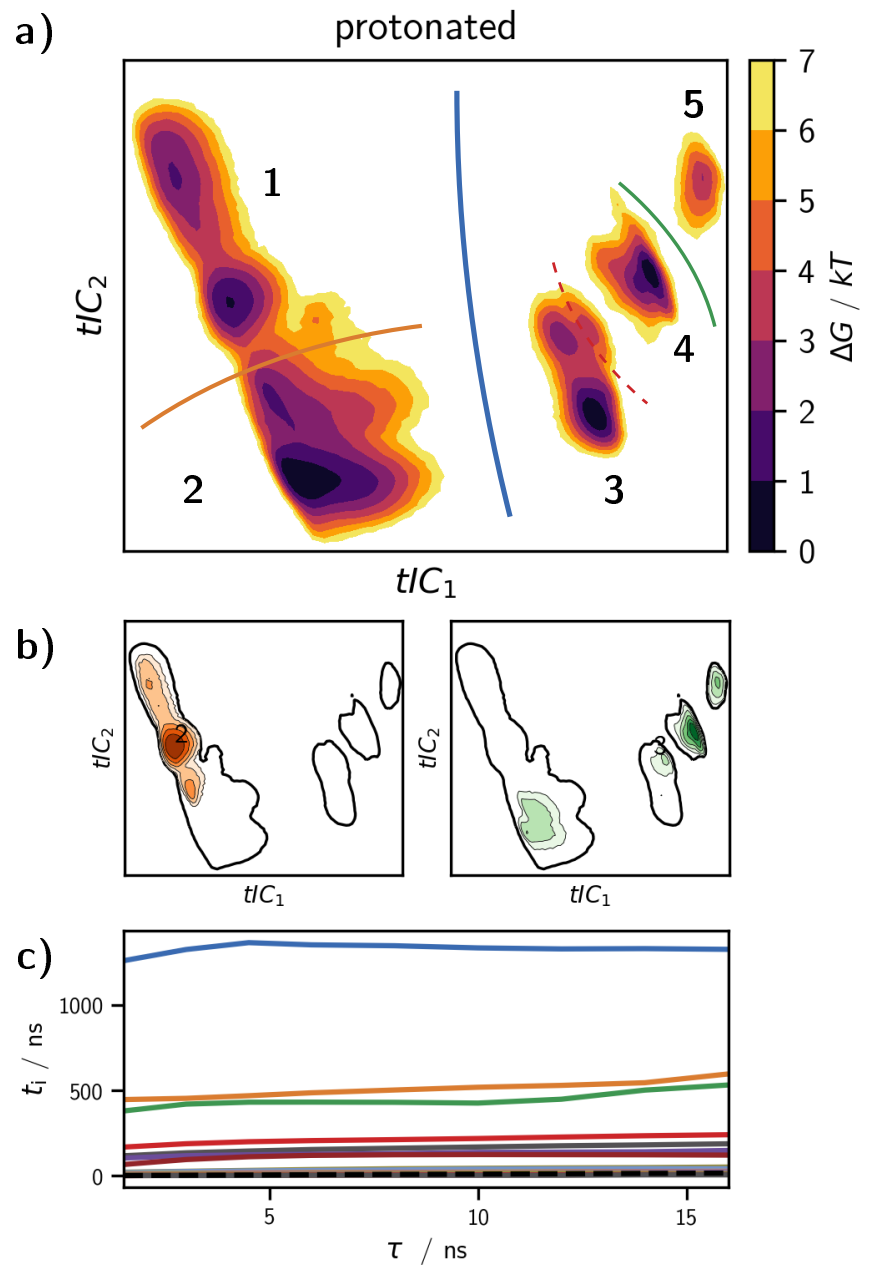

Figure 7: Core-set Markov model of the conformational dynamics of protonated holo-langerin. a) Free energy surfaces from the $2 \mathrm{D}$ projections of protonated holo-langerin trajectories onto the first two tICs. Solid lines: transition regions between the five meta-stable states connected by the four slowest dynamic processes. b) Projections of cluster $G$ (green) and $\mathrm{O}$ (orange) into the space of the first two tICs. c) Implied time scales of the core-set Markov model. The colors of the processes match the transition regions drawn into a).

We then identified 22 core-sets in the space of the first six tICs using common-nearest-neighbors clustering (40-42), and used them to construct a core-set Markov model. The implied time-scale test shows that the timescales of our core-set Markov model are independent of the lag time $\tau$ indicating a very small discretization error and thus a high-quality Markov model (Fig. 7c). The slowest dynamic process occurs on a timescale of about $1.3 \mu \mathrm{s}$ and corresponds to changes in the local conformations of E261 and its hydrogen bond pattern. It thus separates the confor- 
mations of cluster $\mathrm{G}$ (green) and cluster $\mathrm{O}$ (orange) along the blue barrier in Fig. 7a. Note that all conformations in which the K257s-D308s hydrogen bond is formed alongside H294s-E261s are located on the right-hand side of this barrier (see SI). The fact that we find some structures that have originally been assigned to the $\mathrm{G}$ (green) conformation on the left-hand side of the barrier is likely due to the insufficient separation of long-lived conformations in the principal component space (Fig. 7b). Next, protonated langerin has two slow timescales that occur at about $500 \mathrm{~ns}$. One process describes transitions between the closed-loop conformations in region 1 and conformations in which the distance between the long and the short-loop is larger in region 2. The other process represents a transition between conformations in which the backbone-orientation of N291 forbids the $\mathrm{N} 292 \mathrm{~m}-\mathrm{A} 289 \mathrm{~m}$ hydrogen bond giving rise to a distortion of the long-loop (region 5) and the conformations in which the $\mathrm{N} 292 \mathrm{~m}-\mathrm{A} 289 \mathrm{~m}$ hydrogen bond is possible (regions 3 and 4). The dashed barrier marks transitions to more open short-loop forms occurring on a timescale of $210 \mathrm{~ns}$.

In summary, conformations in which the K257-D308 hydrogen bond is formed are separated from the alternative $\mathrm{O}$ (orange) conformation by a rare transition that occurs on a timescale of $1.3 \mu \mathrm{s}$. Within the right-hand side of the barrier in Fig. 7a the $\mathrm{G}$ (green) conformation is at least stable on a timescale of $200 \mathrm{~ns}$. This is likely sufficient to enable the escape of the $\mathrm{Ca}^{2+}$-ion from the binding pocket. A core-set Markov model of neutral holo-langerin is reported in the SI.

To directly probe how the stability of the $\mathrm{Ca}^{2+}$-bound state of the protein depends on the protonation state and on the conformation of langerin, we used constant-velocity steeredMD experiments (49-51). In these simulations, a force that increases linearly with time is applied to the $\mathrm{Ca}^{2+}$-atom (Fig. 8a), and the opposing force (i.e. the resistance against this pulling force) is measured. At a certain maximum force the ionic bonds between the $\mathrm{Ca}^{2+}$-atom and the coordinating residues rupture and the $\mathrm{Ca}^{2+}$-ion leaves the binding pocket. In the computer experiment, this is marked by a sudden drop in the opposing force (Fig. 8c). The rupture force is a rough measure for the free-energy difference to the transition state $\Delta G^{\ddagger}$. The rational is that a deeper free-energy minimum of the $\mathrm{Ca}^{2+}$-bound state is associated with a steeper slope to the transition state, and the rupture force, reflecting the maximal slope, reports on the stability of the $\mathrm{Ca}^{2+}$-bound state (52, 53). We chose the pulling rate such that the rupture events are observed after several nanoseconds. This ensures, that the system has enough time to adjust to the pulling, but also that the initial starting conformation is preserved to some degree.

For each system, we conducted 40 steered-MD simu-
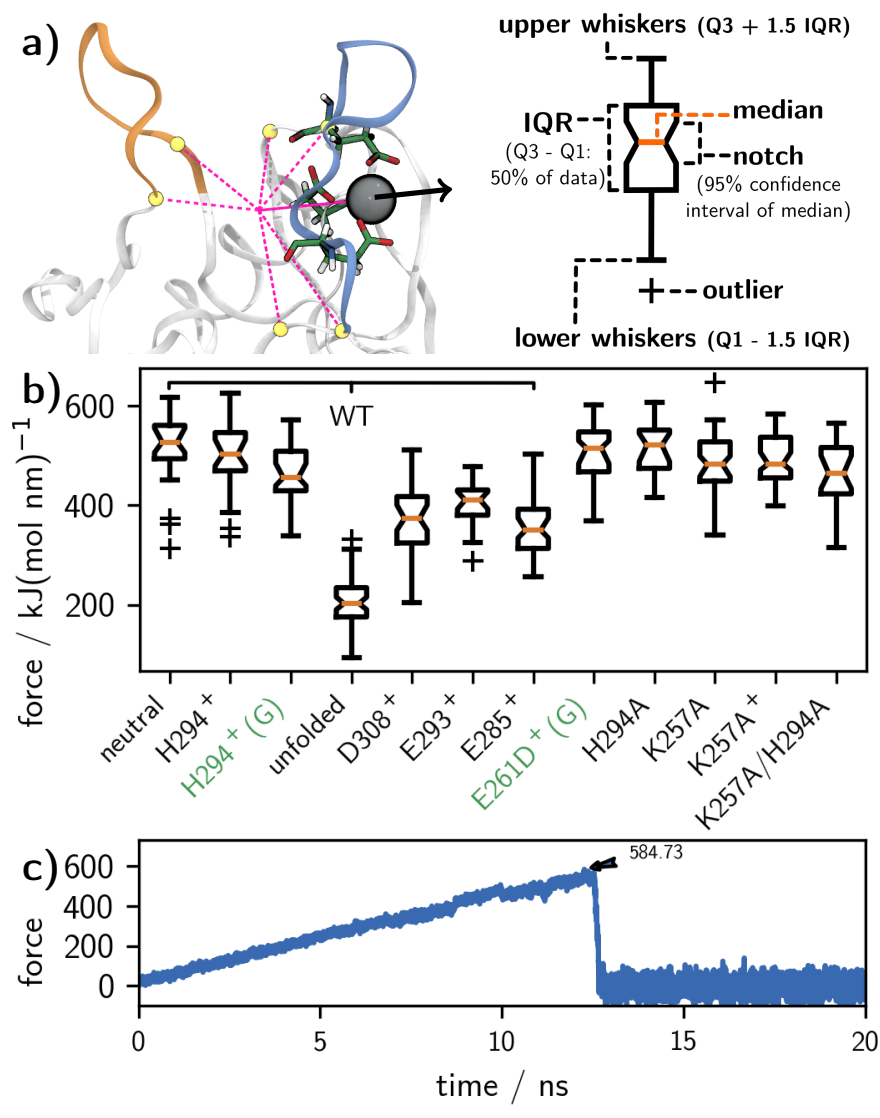

Figure 8: a) Pull coordinate defined as the distance vector between $\mathrm{Ca}^{2+}$ and the center-of-mass of the $\mathrm{C}_{\alpha}$-atoms of residues 257, 264, 281, 282, 293, and 294. b) Maximal pulling force observed acting on $\mathrm{Ca}^{2+}$ during simulations of langerin in various states as notched box representation. The orange line represents the median, while the box enframes the interquartile range. The box notches indicate the $95 \%$ confidence interval on the median. Points lying beyond 1.5 times the edges of the box are regarded as outliers (+), and the whiskers mark the data range without outliers. c) Example for a force trajectory with a rupture event at about 12 ns. Maximum force indicated by an arrow.

lations and report the data as notched boxplot in Fig. 8b. Overall, the plot shows that we could determine the median of rupture force with high confidence and hardly any outliers. The rupture force decreases from the neutral to the protonated system $\left(\mathrm{H} 294^{+}\right)$and then further to simulations of the protonated system started in the $\mathrm{G}$ (green) conformation, in which the K257 amine forms a hydrogen bond with the D308 carboxyl group. This decrease is predicted by our mechanism. Note that classical force-fields cannot model instantaneous shifts in the electron density due to the formations of hydrogen bonds. Thus, the rupture force in the $\mathrm{G}$ (green) conformation might actually be somewhat lower. If the $\mathrm{Ca}^{2+}$-coordinating residue D308 is protonated, corresponding to a situation in which the proton is transferred 
from $\mathrm{K} 257$ to $\mathrm{D} 308$, the rupture force is about $150 \mathrm{~kJ} /(\mathrm{mol}$ $\mathrm{nm})$ lower than in the neutral system.

The same is observed when one of the other two $\mathrm{Ca}^{2+}$ coordinating residues is protonated. A drastic reduction in the rupture force is observed, when the experiment is started from a state where the long-loop is unfolded. This is expected, as one of the $\mathrm{Ca}^{2+}$-coordinating residues E285 is removed from the cage of the binding site in this arrangement. The rupture force for the mutant E261D (started from an analogon of the G conformation) and the mutant H294A are in the same range as for the neutral wild-type langerin.

Notably, the rupture forces for K257A mutants are insensitive towards the modeled state of $\mathrm{H} 294$. The binding capability is virtually the same, no matter if $\mathrm{H} 294$ is neutral, protonated or mutated. This substantiates the importance of $\mathrm{K} 257$ to transport a protonation signal to the $\mathrm{Ca}^{2+}$-binding site.

\section{Comparison to experimental data}

The $\mathrm{Ca}^{2+}$-dissociation constant of wild-type langerin at $\mathrm{pH} 7$ is $K_{\mathrm{d}}=105 \pm 15 \mu \mathrm{M}$, and increases to $K_{\mathrm{d}}=800 \pm 150 \mu \mathrm{M}$ at pH 6 (18), as determined by ITC. These dissociation constants correspond to binding free energies of $\Delta G_{\mathrm{pH}} 7=$ $-22.9 \mathrm{~kJ} / \mathrm{mol}$, and $\Delta G_{\mathrm{pH} 6}=-17.8 \mathrm{~kJ} / \mathrm{mol}$ at $T=300 \mathrm{~K}$, yielding a pH sensitivity of $\Delta \Delta G=\Delta G_{\mathrm{pH}} 6-\Delta G_{\mathrm{pH}} 7=5.1$ $\mathrm{kJ} / \mathrm{mol}$ (Fig. 9). By contrast the dissociation constant of the H294A mutant, in which the $\mathrm{pH}$ sensor $\mathrm{H} 294$ is removed, are $K_{\mathrm{d}}=35 \pm 15 \mu \mathrm{M}$ at pH $7\left(\Delta G_{\mathrm{pH} 7}=-25.6 \mathrm{~kJ} / \mathrm{mol}\right)$, and $K_{\mathrm{d}}=125 \pm 5 \mu \mathrm{M}$ at pH $6\left(\Delta G_{\mathrm{pH} 6}=-22.4 \mathrm{~kJ} / \mathrm{mol}\right)$, corresponding to a reduced $\mathrm{pH}$ sensitivity of $\Delta \Delta G=3.2$ $\mathrm{kJ} / \mathrm{mol}$ (18) (Fig. 9). Our mechanism so far explains the $\mathrm{pH}$ sensitivity due to the $\mathrm{pH}$ sensor $\mathrm{H} 294$. The fact that the H294A mutant exhibits a residual $\mathrm{pH}$-sensitivity indicates that langerin has a second $\mathrm{pH}$ sensor.

To convince ourselves of the robustness of these results, we remeasured the dissociation constants of wild-type langerin (see SI). We obtained $K_{\mathrm{d}}=113 \pm 14 \mu \mathrm{M}$ at $\mathrm{pH} 7$ $\left(\Delta G_{\mathrm{pH}} 7=-22.6 \mathrm{~kJ} / \mathrm{mol}\right)$, and $K_{\mathrm{d}}=802 \pm 150 \mu \mathrm{M}$ at $\mathrm{pH}$ $6\left(\Delta G_{\mathrm{pH} 6}=-17.8 \mathrm{~kJ} / \mathrm{mol}\right)$, yielding a $\mathrm{pH}$ sensitivity of $\Delta \Delta G=4.8 \mathrm{~kJ} / \mathrm{mol}$ (Fig. 9). This is in excellent agreement with our previous results.

Four residues are central to our mechanism: H294, K257, D308, and E261. D308 directly coordinates to $\mathrm{Ca}^{2+}$ and is therefore not a suitable candidate for site-directed mutagenesis. In contrast to $\mathrm{H} 294 \mathrm{~A}$, the $\mathrm{pH}$-sensitivity of K257A could not be determined because the protein precipitated at pH 7. However, both mutants have a higher $\mathrm{Ca}^{2+}$-affinity than wild-type langerin at $\mathrm{pH} 6$, which previously could not be explained. The overall higher $\mathrm{Ca}^{2+}$-affinity in the K257A mutant is predicted by our mechanism, because the K257-D308 hydrogen bond that destabilizes the $\mathrm{Ca}^{2+}$ - coordination cannot be formed in the absence of the K257 side-chain. The H294A mutant has the K257 side-chain, and the conformation in which K257 is in the vicinity of D308 (Fig. 6) can in principle be formed. However, in our simulations of H294A we find that the K257 side-chain is in the vicinity of the D308 side-chain in only $1.7 \%$ of the simulated structures, which might explain the higher $\mathrm{Ca}^{2+}$-affinity of the H294A mutant (see SI).

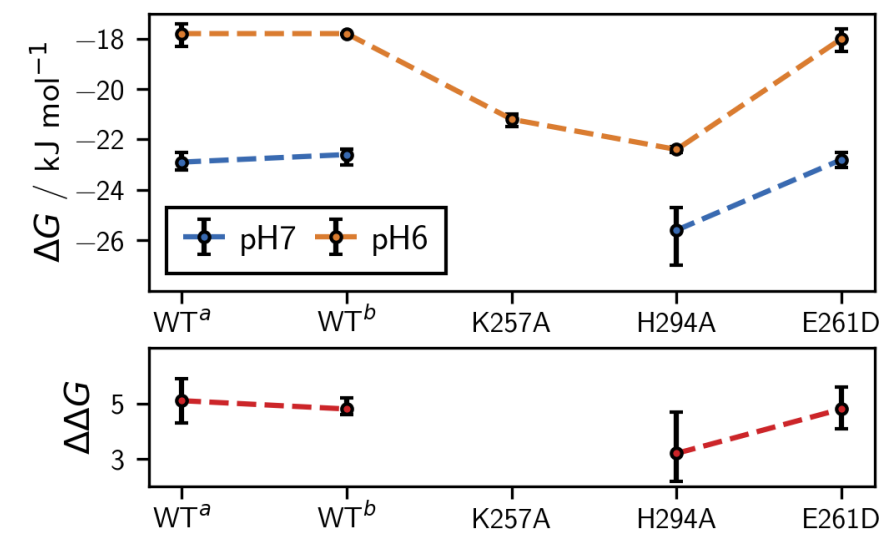

Figure 9: $\mathrm{Ca}^{2+}$-binding free energies under standard conditions in $\mathrm{kJ} / \mathrm{mol}$, calculated as $\Delta G=-R T \ln \left(K_{\mathrm{d}}\right)$, where $R=8.314 \mathrm{~J} /(\mathrm{K} \mathrm{mol})$ is the gas constant, $T=300 \mathrm{~K}$ is the temperature, and $K_{\mathrm{d}}$ in units of $\mathrm{mol} / \mathrm{L}$ are the experimentally determined dissociation constants. Measurements at $\mathrm{pH}$ 6 (blue) and pH 7 (orange) with experimental uncertainties indicated with errorbars and $\mathrm{pH}$-sensitivities in $\mathrm{kJ} / \mathrm{mol}$ calculated as $\Delta \Delta G=\Delta G_{\mathrm{pH} 6}-\Delta G_{\mathrm{pH}} 7$ (red).

Besides H294 and K257, residue E261 is important for the stabilization of the $\mathrm{G}$ (green) conformation, which is responsible for lowering the $\mathrm{Ca}^{2+}$-affinity. However, it also stabilizes the cluster $\mathrm{O}$ (orange), which is not expected to increase the $\mathrm{Ca}^{2+}$-affinity, because $\mathrm{K} 257$ forms a hydrogen bond with E261 rather than with D308 in this conformation. We therefore predicted that mutating E261 has little effect on the $\mathrm{pH}$-sensitivity. We measured the $\mathrm{Ca}^{2+}$-dissociation constants for the E261D mutant at $\mathrm{pH} 6$ and $\mathrm{pH} 7$ by ITC (see $\mathrm{SI}$ ), and the results confirm our prediction. The dissociation constants of the E261D mutant are $K_{\mathrm{d}}=108 \pm 11 \mu \mathrm{M}$ at $\mathrm{pH} 7\left(\Delta G_{\mathrm{pH}} 7=-22.8 \mathrm{~kJ} / \mathrm{mol}\right)$, and $K_{\mathrm{d}}=742 \pm 141 \mu \mathrm{M}$ at $\mathrm{pH} 6\left(\Delta G_{\mathrm{pH} 6}=-18.0 \mathrm{~kJ} / \mathrm{mol}\right)$, yielding a pH sensitivity of $\Delta \Delta G=4.8 \mathrm{~kJ} / \mathrm{mol}$ (Fig. 9).

\section{Long-loop unfolding}

So far, our mechanisms explains how $\mathrm{Ca}^{2+}$ is destabilized in the binding pocket of holo-langerin. However if the proton is transferred from K257 to D308, the mechanism also has profound effects on apo-langerin. In holo-langerin the long-loop is stabilized in a well-defined conformation 
a) 1
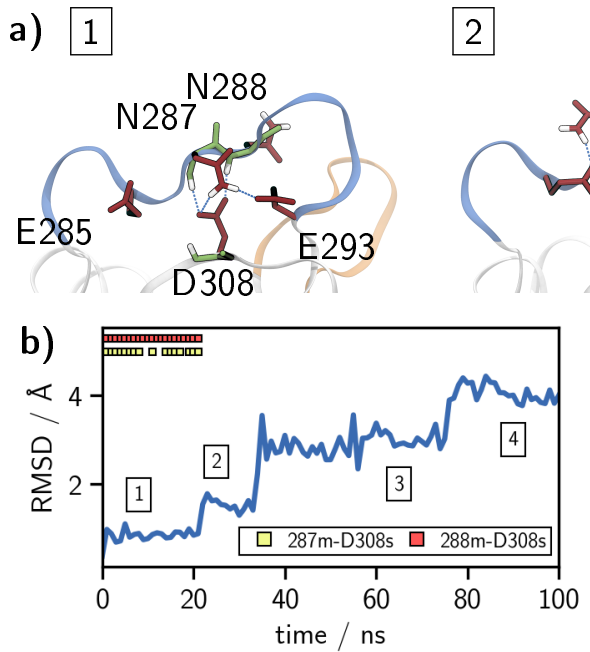

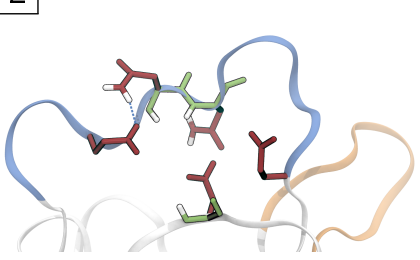

c)

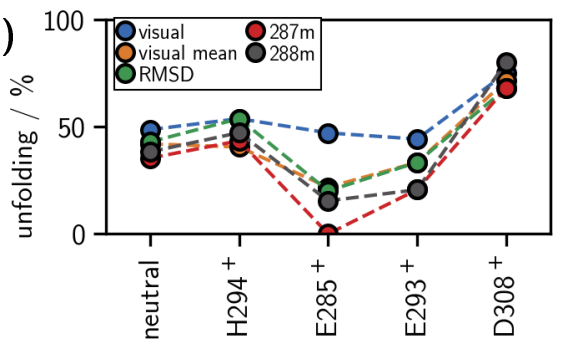

3

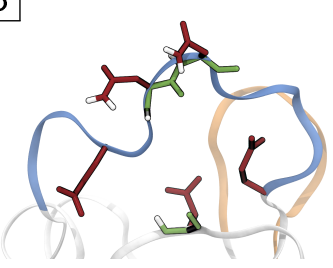

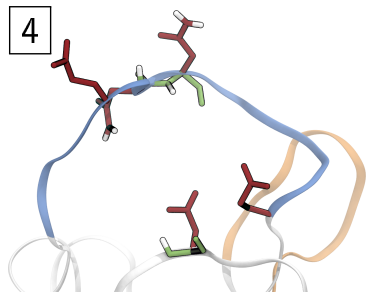

d)

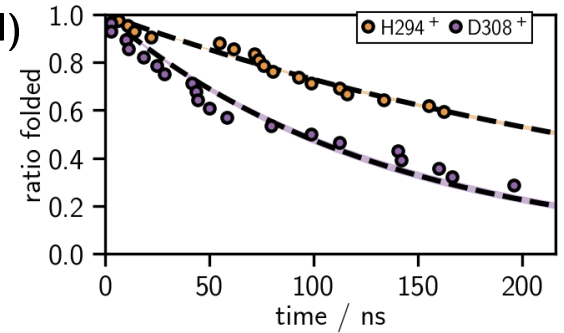

Figure 10: a) Long-loop unfolding in apo-langerin with example structures for a 1 fully folded, 2 intermediate, 3 partially unfolded, and 4 fully unfolded state. b) Example trajectory of the long-loop $\mathrm{C}_{\alpha}-\mathrm{RMSD}, 22$ ns: intermediate state, 30 ns: unfolding event c) Percentage of unfolded trajectories within $220 \mathrm{~ns}$ determined by: last folded frame (visual), mean of last folded and first unfolded frame (visual mean), RMSD $>0.2 \mathrm{~nm}$, and hydrogen bonds N287m-D308s (287m) and N288m-D308s (288m). d) Decay plot of folded trajectories (last folded frame) and exponential fit (dashed line $\pm \sigma$ ), H294': H294 protonated, D308 ${ }^{+}$: H294 and D308 protonated.

(folded long-loop conformation) by E285 which coordinates to $\mathrm{Ca}^{2+}$. In apo-langerin this interaction is not possible, and the long-loop spontaneously unfolds in our simulations. Similar long-loop unfolding has been observed in the crystal structures of other C-type lectins, like tetranectin (54), TC14 (55) or MBP (56). To estimate the unfolding rate we conducted 30 to 60 simulations (see SI) for each of the following protonation states of apo-langerin: neutral, H294 protonated, H294 and E285 protonated, H294 and E293 protonated, and H294 and D308 protonated, each of them started in the folded conformation. In four of the five protonation states 44 to $54 \%$ of all trajectories unfold within $220 \mathrm{~ns}$ simulation time, as determined by visual inspection (Fig. 10c, blue dots). The carboxyl group D308 is critical for the stabilization of the folded loop conformation in the absence of $\mathrm{Ca}^{2+}$ by forming hydrogen bonds with the $\mathrm{N} 287$ side-chain, as well as with the backbone amide-hydrogen of N287 and N288 (Fig. 10a). If D308 is protonated, all three hydrogen bonds are much weaker, and consequently the long-loop unfolds at a higher rate (75\% within $220 \mathrm{~ns}$ ).

Long-loop unfolding often occurs via an intermediate conformation, in which the hydrogen bonds with the backbone amides of N287 and N288 are broken, while the hydrogen bond to the N287 side-chain is still possible. In this intermediate form the loop is more flexible than in the fully folded state, but the characteristic turns in the loop backbone are still largely present, and we observe refolding to the fully folded state in some of the trajectories. The transition to the fully unfolded conformation occurs when one or more of the backbone torsion angles in the long-loop rotate, and the hydrogen bond between the side-chains of D308 and N287 breaks. This transition is irreversible on the timescale of our simulations.

To corroborate our visual analysis of the simulation end points, we determined the time of the unfolding event by four additional criteria: the mean between last fully folded frame and first fully unfolded frame determined by visual inspection, the $\mathrm{C}_{\alpha}$-RMSD of the long-loop residues exceeds $0.2 \mathrm{~nm}$, and breaking of the hydrogen bonds between the D308 carboxyl group or the backbone amide-hydrogen of N287 and N288. All four criteria confirm the first analysis (Fig. 10c). If E285 is protonated a hydrogen bond between the protonated carboxyl group of E285 and the unprotonated carboxyl group of D308 stabilizes a partially folded loop structure, such that for some criteria we observe even fewer unfolding events than by the simple visual analysis for this system. We determined the half-life periods $t_{1 / 2}$ of the folded states from the decay plots of the folded trajectories (see SI). Independent of the criterion, the decay is fastest, when D308 is protonated. In particular unfolding is over twice as fast if D308 is protonated than if only H294 is protonated $\left(t_{1 / 2}=218\right.$ versus $93 \mathrm{~ns}$, Fig. 10d). Some of the decays deviate from a single-exponential decay, hinting at a more complex underlying unfolding mechanism.

Since the folded conformation binds $\mathrm{Ca}^{2+}$ much more strongly than the unfolded conformation (Fig. 8), the equilibrium between folded and unfolded long-loop is critical for the overall $\mathrm{Ca}^{2+}$-affinity. Thus, the protonation of D308 
has a two-fold effect: First, it destabilizes the $\mathrm{Ca}^{2+}$-ion in the binding pocket. Second, after the $\mathrm{Ca}^{2+}$-ion has left the binding pocket, it destabilizes the folded loop conformation and thereby reduces the likelihood of $\mathrm{Ca}^{2+}$-rebinding.

\section{The second $\mathrm{pH}$ sensor}

In the ITC experiments the H294A mutant exhibits a $\mathrm{pH}$ sensitivity of $\Delta G=3.2 \mathrm{~kJ} / \mathrm{mol}$ even though the $\mathrm{pH}$ sensor H294 is missing (18). This suggests that langerin has a second $\mathrm{pH}$ sensor. To convince ourselves that this residual $\mathrm{pH}$ sensitivity is indeed due to a second $\mathrm{pH}$ sensor, we checked whether $\mathrm{K} 257$ forms another potentially $\mathrm{pH}-$ sensitive hydrogen bond in the H294A mutant which could replace the $\mathrm{pH}$-sensitive $\mathrm{K} 257-\mathrm{H} 294$ hydrogen bond and explain the residual $\mathrm{pH}$ sensitivity. In our simulations of the H294A mutant, K257 does not form any highly populated hydrogen bond. With $13 \%$ population the hydrogen bond between the side-chain of K257 and the main-chain carbonyl group of E293 is the most frequently formed hydrogen bond. However, in wild-type langerin it is formed with the same frequency. All other hydrogen bonds of K257 are populated with less than $5 \%$. Thus, the experimentally determined pH-sensitivity in the H294A mutant does indeed indicate that wild-type langerin has a second $\mathrm{pH}$ sensor.

There are two possible mechanisms to explain the residual pH-sensitivity. First, langerin could have a second allosteric $\mathrm{pH}$ sensor that, similar to $\mathrm{H} 294$, is activated by protonation from the surrounding solvent prior to the dissociation of $\mathrm{Ca}^{2+}$. Second, the carboxyl groups of the $\mathrm{Ca}^{2+}$-coordinating residues E285, E293, and D308 could form a dyad with an effective $\mathrm{p} K_{\mathrm{a}}$ that makes it sensitive to a $\mathrm{pH}$ change form 7 to 6. That is, after initial dissociation of $\mathrm{Ca}^{2+}$, one of the coordinating residues (Fig. 2d) is protonated and the protonated state is stabilized as a hydrogen bond to an unprotonated carboxyl group (57). We first discuss the possibility of a second allosteric $\mathrm{pH}$ sensor before investigating whether a dyad is possible.

$\mathrm{H} 229$ is the only other histidine residue in langerin. It is solvent exposed and will indeed be protonated when the $\mathrm{pH}$ changes from 7 to 6 . However, $\mathrm{H} 229$ is located far away from the $\mathrm{Ca}^{2+}$-binding site which makes an allosteric influence on the $\mathrm{Ca}^{2+}$-binding affinity unlikely (Fig. 1). This is further corroborated by the previously published mutual information analysis of the allosteric network in langerin and by chemical shift perturbation experiments (18). In the extended simulation data set used for this study, protonation of H229 has a local effect on the "lower" protein region including the $\alpha_{1}$-helix, but these conformational shifts are well separated from the $\mathrm{Ca}^{2+}$-binding site. We therefore exclude $\mathrm{H} 229$ as a potential $\mathrm{pH}$-sensor.

Other candidates for allosteric $\mathrm{pH}$ sensors are aspartic and glutamic acids, whose $\mathrm{p} K_{\mathrm{a}}$ (in water at $25^{\circ} \mathrm{C} 4.15$ for $\mathrm{E}$ and 3.71 for D) (28) can be shifted by several $\mathrm{p} K_{\mathrm{a}}$ units by the local environment in the protein, such that their carboxyl groups could become sensitive to a $\mathrm{pH}$ change from 7 to 6 (58). Apart from the $\mathrm{Ca}^{2+}$-coordinating residues E285, E293, and D308, langerin has nine aspartic or glutamic acids. Using PROPKA $3.1(59,60)$, we calculated the distribution of the $\mathrm{p} K_{\mathrm{a}}$-values for these residues in holo-langerin in the neutral and the H294-protonated state, as well as for apo-langerin in the neutral and the H294-protonated state. The distributions are based on 10,000 to 30,000 structures extracted from the simulations of the corresponding systems, and are reported along with the mean and the standard deviation in the SI. The mean $\mathrm{p} K_{\mathrm{a}}$-value of all tested residues is below 5.0, and none of the distributions reaches into the critical region between $\mathrm{pH} 6$ and 7, indicating that none of them acts as $\mathrm{pH}$ sensor. We therefore conclude that the residual $\mathrm{pH}$-sensitivity in langerin is not generated by a second allosteric $\mathrm{pH}$-sensor.

PROPKA 3.1 can detect the coupling between two carboxyl groups that are in close vicinity. It returns two alternative $\mathrm{p} K_{\mathrm{a}^{-}}$-values. In alternative $a$, one carboxyl group is protonated first and stabilized by the second (unprotonated) carboxyl group, in alternative $b$ the situation is reversed. Fig. 11a shows the $\mathrm{p} K_{\mathrm{a}}$-distribution of the $\mathrm{Ca}^{2+}$ coordinating residues E285, E293, and D308 as well as the $\mathrm{p} K_{\mathrm{a}}$-distribution of $\mathrm{H} 294$ for apo-langerin in the neutral and the H294-protonated state. No coupling between E285, E293, and D308 was detected by PROPKA 3.1. Their mean $\mathrm{p} K_{\mathrm{a}}$-value is below 5.0, and none of the distributions reaches into the critical region between $\mathrm{pH} 6$ and 7. By contrast, the mean $\mathrm{p} K_{\mathrm{a}}$-values of $\mathrm{H} 294$ are about 6 in the neutral and the $\mathrm{H} 294$-protonated state, and the $\mathrm{p} K_{\mathrm{a}}$-distributions have a large overlap with the critical region between pH 6 and 7 . Thus, from these simulations one would conclude, that langerin does not have a protonatable dyad in the $\mathrm{Ca}^{2+}$-binding pocket, and that only $\mathrm{H} 294$ is sensitive to a $\mathrm{pH}$ change from 7 to 6 .

However, in the neutral and the H294-protonated state, the carboxyl group of the $\mathrm{Ca}^{2+}$-coordinating residues are negatively charged and repel each other, making structures in which the two carboxyl groups are close enough to potentially stabilize a protonation unlikely. We therefore also calculated the $\mathrm{p} K_{\mathrm{a}}$-distribution for the following protonation states of apo-langerin: H294 and E285 protonated (Fig. 11b), H294 and E293 protonated (Fig. 11c), and H294 and D308 protonated (Fig. 11d). For these protonation states, substantial coupling between the $\mathrm{Ca}^{2+}$-coordinating residues is detected. D308 and E285 couple in $74 \%$ of all structure if E285 is protonated, and in $56 \%$ of all structures if D308 is protonated. When E293 is protonated, E293 and 
D308 couple in $27 \%$ of all structures.

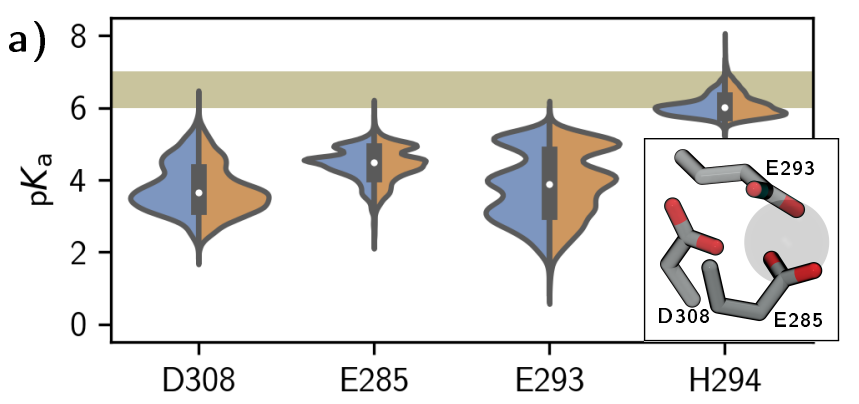

alternative a
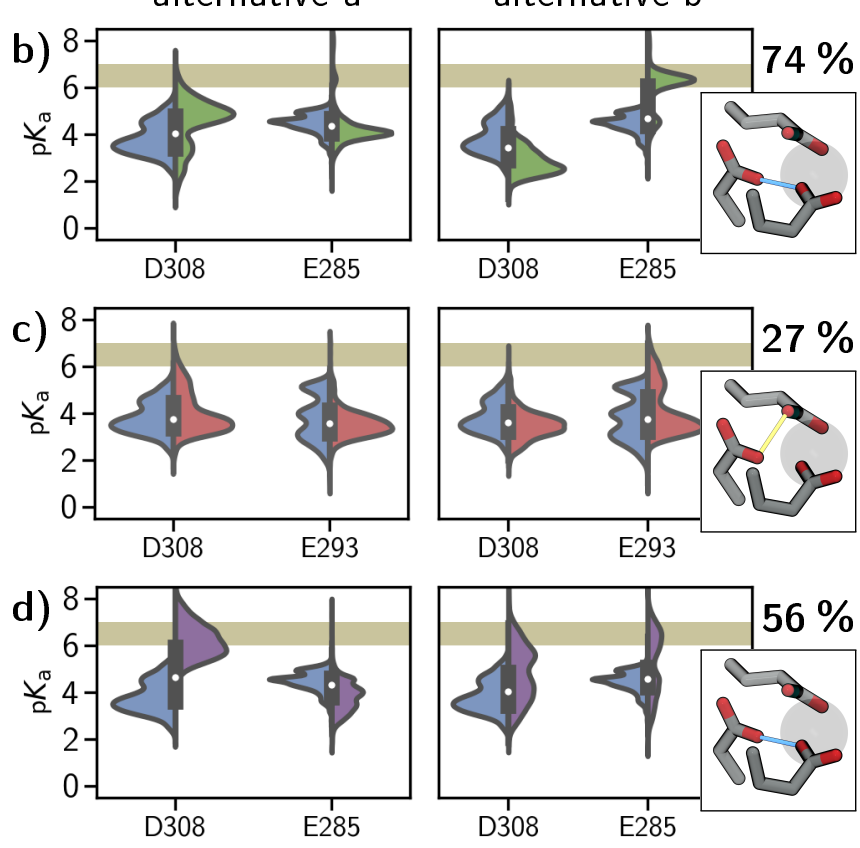

Figure 11: $\mathrm{p} K_{\mathrm{a}}-$ Value distributions (all frames considered) calculated with PROPKA 3.1 for a) the neutral apo- (blue) and the H294 protonated (orange) system. Distributions for residues involved in coupling in b) neutral versus E285 protonated (green), c) neutral versus E293 protonated (red), and d) neutral versus D308 protonated (purple). Alternative distributions due to the coupling left and right. Percentages of coupling frames are placed over the binding site illustrations.

These couplings give rise to a strong shift of the $\mathrm{p} K_{\mathrm{a}}$ distributions compared to neutral apo-langerin. We report the distributions of both $\mathrm{p} K_{\mathrm{a}}$-estimates, which should be interpreted as limiting cases of the true distribution. If D308 is protonated, the $\mathrm{p} K_{\mathrm{a}}$-distributions of $\mathrm{D} 308$ for both limiting cases reach well into the critical region between $\mathrm{pH}$ 6 and 7, and for alternative $a$ we obtain a mean $\mathrm{p} K_{\mathrm{a}}$-value in coupling frames of $6.4 \pm 0.7$ (Fig. 11d). If E285 is protonated, the coupling to D308 in alternative $b$ yields a mean $\mathrm{p} K_{\mathrm{a}}$-value of $6.2 \pm 0.6$ for E285, and the corresponding distribution of all frames is almost centered on the critical region between pH 6 and 7 (Fig. 11b). The effect is not as strong, if E293 is protonated (Fig. 11b). For alternative $a$ the $\mathrm{p} K_{\mathrm{a}}$-distribution of $\mathrm{D} 308$ reaches slightly into the region between $\mathrm{pH} 6$ and 7 , and for alternative $b$ the $\mathrm{p} K_{\mathrm{a}^{-}}$ distribution of E293 reaches into this region. However, the corresponding $\mathrm{p} K_{\mathrm{a}}$-values, $5.2 \pm 0.7$ and $5.5 \pm 0.7$, are clearly lower than those for the coupling between D308 and E285.

These results show that in the absence of $\mathrm{Ca}^{2+}, \mathrm{D} 308$ and E285 can form a protonated dyad with an effective $\mathrm{p} K_{\mathrm{a}}$ that is likely high enough to sense a $\mathrm{pH}$ change from 6 to 7. We therefore believe that the second $\mathrm{pH}$ sensor that is active in the H294A mutant is the dyad between D308 and E285. In wild-type langerin the $\mathrm{pH}$ sensor $\mathrm{H} 294$ and this dyad would amplify each other: the K257-D308 hydrogen bond increases the probability that D308 is protonated, and, after $\mathrm{Ca}^{2+}$ has escaped, the protonated D308 is stabilized by the D308-E285 dyad. Constant-pH simulations (61-63) or $\mathrm{QM} / \mathrm{MM}$ simulations $(64,65)$ could be used to verify whether D308 and E285 indeed form a dyad and constitute the second $\mathrm{pH}$ sensor.

Note that the conformational fluctuations in the $\mathrm{Ca}^{2+}$ binding pocket give rise to large fluctuations in the instantaneous $\mathrm{p} K_{\mathrm{a}}$-value (Fig. 11) with some distributions covering more than six $\mathrm{p} K_{\mathrm{a}}$ units. Thus, knowing the underlying conformational distribution is essential for a reliable estimate of the overall $\mathrm{p} K_{\mathrm{a}}$-value.

\section{Comparison to other C-type lectins}

To gain insight into whether the proposed mechanism for the $\mathrm{pH}$ sensitivity is found in other C-type lectins, we compared the sequences of human langerin to mouse langerin and to human variants of 15 related C-type lectins (SI Fig. 31). All 16 proteins exhibit the typical C-type-lectin fold, as evidenced by crystal structures (SI Fig. 32). The residues D308 and E285, which form the proposed second $\mathrm{pH}$ sensor, are highly conserved. However, one should be careful to interpret this as evidence for a conserved second $\mathrm{pH}$-sensor, because these residues are also essential for the coordination of $\mathrm{Ca}^{2+}$ and might be conserved for this reason.

The H294-K257 motif, the primary $\mathrm{pH}$ sensor in langerin, is not conserved in our sequence alignment. Thus, the proposed mechanism for the $\mathrm{pH}$ sensitivity of the $\mathrm{Ca}^{2+}$ affinity via $\mathrm{H} 294$ protonation does not seem to be the most widespread mechanism to sense a change in the environment in C-type lectins. But the sequence alignment points to possible other mechanisms for sensing a change in the environment.

The selectines P-, E-, and LSECtin share the lysine K257 with langerin in the same position. Additionally, the preceding threonine T256 in langerin is replaced by 
an arginine in these three proteins, while $\mathrm{H} 294$ is replaced by an aspartic acid. Note that in LSECtin $\mathrm{Ca}^{2+}$-affinity increases if the $\mathrm{pH}$ decreases (27). It is however unclear whether this reversed $\mathrm{pH}$ sensitivity is brought about by the change of the H294-K257 motif. Other lysine residues in the short-loop in comparable positions as K257 in langerin can also be found in MCL, lung surfactant protein (SP), CD23a, Endo180, and MMR.

H294 only appears in human and mouse langerin, and is replaced by aspartic acid in most of the other C-type lectins. Instead we find a $\mathrm{Ca}^{2+}$-cation in the position where langerin has the H294-K257 hydrogen bond in 6 out of 15 lectins in our analysis (ASGPR, MBP, DCSIGN, DCSIGNR, SP, SR). This $\mathrm{Ca}^{2+}$-cation would be partially solvent exposed even when a large entity (such as a pathogen) is bound to the C-type lectin (SI Fig. 32). One therefore might speculate that these lectins do not sense a change in $\mathrm{pH}$ but rather a sense in $\mathrm{Ca}^{2+}$ concentration.

Several C-type lectins have histidines in other positions close to the $\mathrm{Ca}^{2+}$ binding site, which might act as $\mathrm{pH}$ sensors via a different mechanism. As already mentioned, ASGPR has a histidine residue that is close to the $\mathrm{Ca}^{2+}$ in the primary $\mathrm{Ca}^{2+}$ binding site and thereby acts as $\mathrm{pH}$ sensor. Furthermore, dectin- 2 and MMR have a histidine residue as a neighbor to a $\mathrm{Ca}^{2+}$-coordinating residue, and Endo180 and MCL have histidines at the beginning of the long-loop. Whether these histidines act as $\mathrm{pH}$ sensors can be tested by mutating the histidine residue and measuring the pH-sensitivity of the $\mathrm{Ca}^{2+}$ affinity and of the carbohydrate affinity. Once a residue is a confirmed as a $\mathrm{pH}$ sensor, the approach presented in this contribution can be used to propose a molecular mechanism for the $\mathrm{pH}$ sensitivity.

\section{Conclusion}

We have described the consequences of a $\mathrm{H} 294$ protonation in langerin and its implications for its biological function as an endocytic pattern recognition receptor. When langerin enters the acidic environment of an endosome, it releases its $\mathrm{Ca}^{2+}$ co-factor and subsequently its pathogenic cargo, triggered by a moderate change in $\mathrm{pH}$. The $\mathrm{Ca}^{2+}$-binding site is blocked from direct solvent access by the pathogen, and additionally the $\mathrm{Ca}^{2+}$-coordinating residues have low protonation probabilities in the presence of calcium. Instead, $\mathrm{H} 294$ acts as an accessible site, sensing already a change in $\mathrm{pH}$ from 7 to 6 (18).

In this contribution, we have uncovered a mechanism in which protonation of $\mathrm{H} 294$ perturbs the hydrogen bonded network of the surrounding residues, and alters the conformational ensemble of langerin. A new conformation becomes accessible, in which the protonated K257 side- chain forms a hydrogen bond with the $\mathrm{Ca}^{2+}$-coordinating D308, thereby moving a positive charge into the vicinity of the $\mathrm{Ca}^{2+}$-binding site. This alone can facilitate the $\mathrm{Ca}^{2+}$ release as shown by the reduction in the required force to pull out the ion from its binding site in our steered MD experiments.

The close availability of K257 as a proton source next to the $\mathrm{Ca}^{2+}$-binding site possibly results in a proton transfer to the side-chain of D308. At least it has been shown in a theoretical model, that the neutral form of a lysine-aspartate pair can be favored over the salt-bridge, if the dielectric constant of the medium is low as it can be the case in the environment of a protein (43). Thus, protonation of the initial $\mathrm{pH}$ sensor H294 likely triggers a cascade of events that ensures the unbinding of $\mathrm{Ca}^{2+}: \mathrm{K} 257$ transfers a proton to D308, protonation of D308 competes drastically with $\mathrm{Ca}^{2+}$-binding and, after $\mathrm{Ca}^{2+}$ is expelled, the protonation of D308 is stabilized by a dyad with E285. Protonation of D308 additionally accelerates the unfolding of the long-loop, preventing $\mathrm{Ca}^{2+}$ from rebinding.

For langerin's role as endocytic pattern recognition receptor a fast and irreversible $\mathrm{Ca}^{2+}$ release is essential. On the cell surface, $\mathrm{Ca}^{2+}$ needs to be tightly bound such that the receptor is continuously ready to bind to pathogens. Yet, after endocytosis langerin is probably recycled within minutes $(13,66)$. This leaves little time for the release of the pathogen, which must be preceded by the unbinding of $\mathrm{Ca}^{2+}$. The mechanism that we proposed is an elegant solution to these contradicting requirements: the $\mathrm{Ca}^{2+}$-unbinding rate is increased by the K257-D308 hydrogen bond, and after the initial $\mathrm{Ca}^{2+}$ release, a transfer of the proton from $\mathrm{K} 257$ to D308 triggers a transition to a conformation to which $\mathrm{Ca}^{2+}$ cannot rebind.

Note that while our results show that the K257-D308 interaction decreases the stability of $\mathrm{Ca}^{2+}$ in the binding pocket and that the protonation of $\mathrm{D} 308$ triggers the longloop unfolding, the transfer of a proton from K257 to D308 is currently an assumption. More work is needed to study the equilibrium between the initial and the end state of the proton transfer. Computationally, this could be tackled by QM/MM calculations $(64,65)$, free-energy calculations with classical force fields, or by constant $\mathrm{pH}$ simulations (61-63).

Another concern is that the point charge $\mathrm{Ca}^{2+}$ model might not capture the energetics of $\mathrm{Ca}^{2+}$-binding accurately enough, because the point charge model does not enforce coordination and neglects polarization effects. In our study, we tried to minimize the influence of these force field effects by analyzing the differences between two protonation states. However, more elaborate $\mathrm{Ca}^{2+}$ models such as reparametrized point-charge models $(67,68)$, multisite 
models (69), or polarizable models (70) are available, and should be used for example for the computation of statespecific $\mathrm{Ca}^{2+}$ binding free-energies.

Our close atomistic inspection of langerin and its conformational shift upon protonation, gives insight into how pH-sensitivity can be incorporated in biological systems. What seemed like a general conformational shift upon protonation in Fig. 3 could be focused to a specific rearrangement of a side chain (K257) to transport the information from the primary $\mathrm{pH}$ sensor (H294) to the allosterically regulated site $\left(\mathrm{Ca}^{2+}\right.$-binding site). Even though the H294-K257 motif is not typical for C-type lectins, many of these proteins exhibit a highly specific $\mathrm{pH}$ sensitivity and have potential $\mathrm{pH}$ sensors in the vicinity of the primary $\mathrm{Ca}^{2+}$-binding site. Our approach can serve as a road map to elucidate the mechanism of $\mathrm{pH}$ sensitivity in these systems.

\section{Experimental procedures}

\section{Molecular dynamics simulations}

We used the software package GROMACS (71-77) in setup and production to simulate the considered systems in the NPT-ensemble (1 bar, $300 \mathrm{~K}$ ) with AMBER99SB-ILDN force-field parameters (78) and the TIP3P water model (79). Prior to production, starting structures were put into a sufficiently large simulation box, solvated, neutralized and equilibrated for several hundred picoseconds. For further details refer to the SI.

\section{Protein expression and purification}

All standard chemicals and buffers used within this work were purchased from Sigma Aldrich (St. Louis, MO, USA) or Carl Roth (Karlsruhe, Germany) if not indicated otherwise.

Human langerin CRD WT and all mutants (amino acids 193-328) were cloned from a codon-optimized langerin gene for bacterial expression (GenScript, Piscataway, NJ, USA) into a pET-28a vector (GenScript, Piscataway, NJ, USA) with His-tag, T7 promoter and Kanamycin resistance. Insoluble expression was performed in E. coli BL21 (ThermoFisher Scienific, Waltham, MA, USA) in LB medium or in isotope-labeled M9 medium at $37{ }^{\circ} \mathrm{C}$. Protein expression was induced by adding $1 \mathrm{mM}$ IPTG. Bacteria were harvested $3-4 \mathrm{~h}$ after induction by centrifugation at $4.000 \mathrm{~g}$ for $30 \mathrm{~min}$. Cell pellets were lysed in lysis buffer $(50 \mathrm{mM}$ Tris, $150 \mathrm{mM} \mathrm{NaCl}, 10 \mathrm{mM} \mathrm{MgCl} 2,0.1 \%$ Tween-20, $\mathrm{pH}$ 8) with $1 \mathrm{mg} \mathrm{mL}^{-1}$ lysozyme and $100 \mu \mathrm{gL} \mathrm{mL}^{-1}$ DNase I (Applichem, Darmstadt, Germany) for at least $3 \mathrm{~h}$ at RT. Inclusion bodies were washed twice with $20-30 \mathrm{~mL}$ lysis buffer and twice with water to remove soluble proteins.
Inclusion bodies were denatured in $20 \mathrm{~mL}$ of denaturation buffer $(6 \mathrm{M}$ guanidinium hydrochloride in $100 \mathrm{mM}$ Tris, $\mathrm{pH} 8$ ) with $0.01 \% \beta$-mercaptoethanol for at least $1 \mathrm{~h}$ at $37^{\circ} \mathrm{C}$ by shaking or overnight at $4^{\circ} \mathrm{C}$ by rotating. After centrifuging $\left(15.000 \mathrm{~g}, 90 \mathrm{~min}, 4^{\circ} \mathrm{C}\right)$, the supernatant was slowly diluted 1:10 with langerin refolding buffer $(0.4 \mathrm{M}$ L-arginine in $50 \mathrm{mM}$ Tris, $20 \mathrm{mM} \mathrm{NaCl}, 0.8 \mathrm{mM} \mathrm{KCl}, \mathrm{pH}$ 7.5) with $1 \mathrm{mM}$ reduced glutathion (GSH) and $0.1 \mathrm{mM}$ oxidized glutathion (GSSG) while stirring at $4{ }^{\circ} \mathrm{C}$ for at least $24 \mathrm{~h}$. The refolded protein solution was dialyzed against $2 \mathrm{~L}$ TBS buffer ( $50 \mathrm{mM}$ Tris, $150 \mathrm{mM} \mathrm{NaCl}, 5 \mathrm{mM} \mathrm{CaCl} 2$ ) and subsequently centrifuged to remove precipitated protein $\left(15.000 \mathrm{~g}, 90 \mathrm{~min}, 4^{\circ} \mathrm{C}\right)$. The supernatant was purified via Ni-NTA agarose affinity chromatography and the elution fractions were pooled and dialyzed against MES $(25 \mathrm{mM}$ MES, $40 \mathrm{mM} \mathrm{NaCl}$, pH 6) or HBS (25 mM HEPES, $150 \mathrm{mM}$ $\mathrm{NaCl}, \mathrm{pH} 7)$ buffer. Precipitated protein was removed by centrifugation $\left(15.000 \mathrm{~g}, 90 \mathrm{~min}, 4^{\circ} \mathrm{C}\right)$ and the supernatant was used for experiments. Note that this procedure varies slightly from the one in our previous paper (18).

\section{ITC measurements}

Isothermal titration calorimetry experiments were performed using a MicroCal iTC200 (Malvern Instruments, Malvern, UK) using either chelex-filtered HBS $(25 \mathrm{mM}$ HEPES, $150 \mathrm{mM} \mathrm{NaCl}, \mathrm{pH} \mathrm{7)}$ or low salt MES buffer ( $25 \mathrm{mM}$ MES, $40 \mathrm{mM} \mathrm{NaCl}, \mathrm{pH}$ 6). The titrant was dissolved in the same buffer as was used for dialysis of the protein sample. Using the iTC200, the titrant $\mathrm{CaCl}_{2}(15 \mathrm{mM}$ stock) was added in defined steps of $1-2.5 \mu \mathrm{L}$ to $80 \mu \mathrm{L}$ protein solution at $298 \mathrm{~K}$ while stirring at $750 \mathrm{rpm}$. The differential heat of each injection was measured and plotted against the molar ratio. The data was fitted to a one-set of sites binding model assuming a Hill coefficient of 1 . Due to the low c-values of the measurements $(c<5)$, the enthalpy could not be determined reliably. See also SI Fig. 29 and 30.

\section{Acknowledgment}

Funded by the Deutsche Forschungsgemeinschaft (DFG, German Research Foundation) under Germany's Excellence Strategy - EXC 2008 - 390540038 - UniSysCat. The authors thank the North-German Supercomputing Alliance (HLRN), the Paderborn Center for Parallel Computing PC ${ }^{2}$ and the ZEDAT of the FU Berlin for computing time. Also funded by the Deutsche Forschungsgemeinschaft (DFG, German Research Foundation) through CRC 765, and DFG RA1944/6-1. We thank the Max Planck Society for support. 


\section{References}

1. Valladeau, J., Duvert-Frances, V., Pin, J. J., DezutterDambuyant, C., Vincent, C., Massacrier, C., Vincent, J., Yoneda, K., Banchereau, J., Caux, C., Davoust, J., and Saeland, S. (1999). The monoclonal antibody DCGM4 recognizes Langerin, a protein specific of Langerhans cells, and is rapidly internalized from the cell surface. European Journal of Immunology 29, 2695-2704.

2. Valladeau, J., Ravel, O., Dezutter-Dambuyant, C., Moore, K., Kleijmeer, M., Liu, Y., Duvert-Frances, V., Vincent, C., Schmitt, D., Davoust, J., Caux, C., Lebecque, S., and Saeland, S. (2000). Langerin, a Novel C-Type Lectin Specific to Langerhans Cells, Is an Endocytic Receptor that Induces the Formation of Birbeck Granules. Immunity 12, 71-81.

3. Zelensky, A. N., and Gready, J. E. (2003). Comparative analysis of structural properties of the C-type-lectinlike domain (CTLD). Proteins: Structure, Function and Genetics 52, 466-477.

4. Zelensky, A. N., and Gready, J. E. (2005). The C-type lectin-like domain superfamily. FEBS Journal 272, 6179-6217.

5. Ng, W. C., Londrigan, S. L., Nasr, N., Cunningham, A. L., Turville, S., Brooks, A. G., and Reading, P. C. (2016). The C-type Lectin Langerin Functions as a Receptor for Attachment and Infectious Entry of Influenza A Virus. Journal of Virology 90, 206-221.

6. van der Vlist, M., de Witte, L., de Vries, R. D., Litjens, M., de Jong, M. A. W. P., Fluitsma, D., de Swart, R. L., and Geijtenbeek, T. B. H. (2011). Human Langerhans cells capture measles virus through Langerin and present viral antigens to CD4+ T cells but are incapable of cross-presentation. European Journal of Immunology 41, 2619-2631.

7. de Witte, L., Nabatov, A., Pion, M., Fluitsma, D., de Jong, M. A. W. P., de Gruijl, T., Piguet, V., van Kooyk, Y., and Geijtenbeek, T. B. H. (2007). Langerin is a natural barrier to HIV-1 transmission by Langerhans cells. Nature Medicine 13, 367-371.

8. de Jong, M. A., Vriend, L. E., Theelen, B., Taylor, M. E., Fluitsma, D., Boekhout, T., and Geijtenbeek, T. B. (2010). C-type lectin Langerin is a $\beta$-glucan receptor on human Langerhans cells that recognizes opportunistic and pathogenic fungi. Molecular Immunology 47, 1216-1225.
9. Hunger, R. E., Sieling, P. A., Ochoa, M. T., Sugaya, M., Burdick, A. E., Rea, T. H., Brennan, P. J., Belisle, J. T., Blauvelt, A., Porcelli, S. A., and Modlin, R. L. (2004). Langerhans cells utilize CD1a and langerin to efficiently present nonpeptide antigens to T cells. Journal of Clinical Investigation 113, 701-708.

10. van Dalen, R., Fuchsberger, F. F., Rademacher, C., van Strijp, J. A., and van Sorge, N. M. (2020). A Common Genetic Variation in Langerin (CD207) Compromises Cellular Uptake of Staphylococcus aureus. Journal of Innate Immunity 12, 191-200.

11. Ribeiro, C. M. S., Sarrami-Forooshani, R., Setiawan, L. C., Zijlstra-Willems, E. M., van Hamme, J. L., Tigchelaar, W., van der Wel, N. N., Kootstra, N. A., Gringhuis, S. I., and Geijtenbeek, T. B. H. (2016). Receptor usage dictates HIV-1 restriction by human TRIM5 $\alpha$ in dendritic cell subsets. Nature 540, 448452.

12. Sorkin, A., and von Zastrow, M. (2002). Signal transduction and endocytosis: close encounters of many kinds. Nature Reviews Molecular Cell Biology 3, 600614.

13. Cote, R., Lynn Eggink, L., and Kenneth Hoober, J. (2017). CLEC receptors, endocytosis and calcium signaling. AIMS Allergy and Immunology 1, 207-231.

14. Onizuka, T., Shimizu, H., Moriwaki, Y., Nakano, T., Kanai, S., Shimada, I., and Takahashi, H. (2012). NMR study of ligand release from asialoglycoprotein receptor under solution conditions in early endosomes. FEBS Journal 279, 2645-2656.

15. Gerasimenko, J. V., Tepikin, A. V., Petersen, O. H., and Gerasimenko, O. V. (1998). Calcium uptake via endocytosis with rapid release from acidifying endosomes. Current Biology 8, 1335-1338.

16. Image of the Zika virus by Thomas Splettstoesser (www.scistyle.com) is licensed under CC BY-SA 4.0 / Adaptation (https://creativecommons.org/licenses/by$\mathrm{sa} / 4.0 /)$.

17. Feinberg, H., Powlesland, A. S., Taylor, M. E., and Weis, W. I. (2010). Trimeric structure of Langerin. Journal of Biological Chemistry 285, 13285-13293.

18. Hanske, J., Aleksić, S., Ballaschk, M., Jurk, M., Shanina, E., Beerbaum, M., Schmieder, P., Keller, B. G., and Rademacher, C. (2016). Intradomain Allosteric Network Modulates Calcium Affinity of the C-Type Lectin Receptor Langerin. Journal of the American Chemical Society 138, 12176-12186. 
19. Stambach, N. S., and Taylor, M. E. (2003). Characterization of carbohydrate recognition by langerin, a C-type lectin of Langerhans cells. Glycobiology 13, 401-410.

20. Loeb, J. A., and Drickamer, K. (1988). Conformational changes in the chicken receptor for endocytosis of glycoproteins. J. Biol. Chem. 263, 9752-9760.

21. Wragg, S., and Drickamer, K. (1999). Identification of Amino Acid Residues That Determine pH Dependence of Ligand Binding to the Asialoglycoprotein Receptor during Endocytosis. Journal of Biological Chemistry 274, 35400-35406.

22. Mullin, N. P., Hall, K. T., and Taylor, M. E. (1994). Characterization of ligand binding to a carbohydraterecognition domain of the macrophage mannose receptor. Journal of Biological Chemistry 269, 2840528413.

23. Guo, Y., Feinberg, H., Conroy, E., Mitchell, D. A., Alvarez, R., Blixt, O., Taylor, M. E., Weis, W. I., and Drickamer, K. (2004). Structural basis for distinct ligand-binding and targeting properties of the receptors DC-SIGN and DC-SIGNR. 11, 591-598.

24. Tabarani, G., Thépaut, M., Stroebel, D., Ebei, C., Vivès, C., Vachette, P., Durand, D., and Fieschi, F. (2009). DC-SIGN neck domain is a pH-sensor controlling oligomerization. SAXS and hydrodynamic studies of extracellular domain. Journal of Biological Chemistry 284, 21229-21240.

25. (2014). NMR evidence for oligosaccharide release from the dendritic-cell specific intercellular adhesion molecule 3-grabbing non-integrin-related (CLEC4M) carbohydrate recognition domain at low $\mathrm{pH}$. FEBS Journal 281, 3739-3750.

26. Mitchell, D. A., Fadden, A. J., and Drickamer, K. (2001). A novel mechanism of carbohydrate recognition by the C-type lectins DC-SIGN and DC-SIGNR. Subunit organisation and binding to multivalent ligands. Journal of Biological Chemistry 276, 2893928945.

27. Powlesland, A. S., Fisch, T., Taylor, M. E., Smith, D. F., Tissot, B., Dell, A., Pöhlmann, S., and Drickamer, K. (2008). A Novel Mechanism for LSECtin Binding to Ebola Virus Surface Glycoprotein through Truncated Glycans. Journal of Biological Chemistry 283, 593602.

28. CRC Handbook of Chemistry and Physics, 87th ed.; Lide, R. D., Ed.; CRC Press: West Palm Beach, 2006.
29. Hyland, L. J., Tomaszek, T. A., and Meek, T. D. (1991). Human immunodeficiency virus-1 protease. 2. Use of $\mathrm{pH}$ rate studies and solvent kinetic isotope effects to elucidate details of chemical mechanism. Biochemistry 30, 8454-8463.

30. Torbeev, V. Y., and Kent, S. B. H. (2012). Ionization state of the catalytic dyad Asp25/25' in the HIV-1 protease: NMR studies of site-specifically $13 \mathrm{C}$ labelled HIV-1 protease prepared by total chemical synthesis. Organic \& Biomolecular Chemistry 10, 5887.

31. Toulokhonova, L., Metzler, W. J., Witmer, M. R., Copeland, R. A., and Marcinkeviciene, J. (2003). Kinetic Studies on $\beta$-Site Amyloid Precursor Proteincleaving Enzyme (BACE). Journal of Biological Chemistry 278, 4582-4589.

32. Huang, Y., Yue, Z., Tsai, C.-C., Henderson, J. A., and Shen, J. (2018). Predicting Catalytic Proton Donors and Nucleophiles in Enzymes: How Adding Dynamics Helps Elucidate the Structure-Function Relationships. The Journal of Physical Chemistry Letters 9, 11791184.

33. Yamazaki, T., Nicholson, L. K., Torchia, D. A., Wingfield, P., Stahl, S. J., Kaufman, J. D., Eyermann, C. J., Hodge, N. C., Lam, P. Y. S., Ru, Y., Jadhav, P. K., Chang, C. H., and Weber, P. C. (1994). NMR and X-ray Evidence That the HIV Protease Catalytic Aspartyl Groups Are Protonated in the Complex Formed by the Protease and a Non-Peptide Cyclic Urea-Based Inhibitor. Journal of the American Chemical Society 116, 10791-10792.

34. Keller, B. G., and Rademacher, C. (2020). Allostery in C-type lectins. Current Opinion in Structural Biology 62, 31-38.

35. Drickamer, K. (1992). Engineering galactose-binding activity into a $\mathrm{C}$-type mannose-binding protein. Nature 360, 183-186.

36. Drickamer, K., and Taylor, M. E. (2015). Recent insights into structures and functions of C-type lectins in the immune system. Current Opinion in Structural Biology 34, 26-34.

37. Li, L., Li, C., Zhang, Z., and Alexov, E. (2013). On the Dielectric "Constant" of Proteins: Smooth Dielectric Function for Macromolecular Modeling and Its Implementation in DelPhi. Journal of Chemical Theory and Computation 9, 2126-2136.

38. Jolliffe, I. T., Principal Component Analysis, 2nd ed.; Springer: New York, 2002. 
39. Scherer, M. K., Trendelkamp-Schroer, B., Paul, F., 49. Pérez-Hernández, G., Hoffmann, M., Plattner, N., Wehmeyer, C., Prinz, J.-H., and Noé, F. (2015). PyEMMA 2: A Software Package for Estimation, Validation, and Analysis of Markov Models. Journal of Chemical Theory and Computation 11, 5525-5542.

40. Keller, B. G., Daura, X., and van Gunsteren, W. F. (2010). Comparing geometric and kinetic cluster algorithms for molecular simulation data. Journal of Chemical Physics 132, 074110.

41. Lemke, O., and Keller, B. G. (2016). Density-based cluster algorithms for the identification of core sets. The Journal of Chemical Physics 145, 164104.

42. Lemke, O., and Keller, B. G. (2018). Common Nearest Neighbor Clustering - A Benchmark. Algorithms 11, 19.

43. Nagy, P. I., and Erhardt, P. W. (2010). Theoretical Studies of Salt-Bridge Formation by Amino Acid Side Chains in Low and Medium Polarity Environments. The Journal of Physical Chemistry B 114, 1643616442.

44. Schütte, C., Noé, F., Lu, J., Sarich, M., and VandenEijnden, E. (2011). Markov state models based on milestoning. Journal of Chemical Physics 134, DOI: $10.1063 / 1.3590108$.

45. Prinz, J.-H., Wu, H., Sarich, M., Keller, B., Senne, M., Held, M., Chodera, J. D., Schütte, C., and Noé, F. (2011). Markov models of molecular kinetics: Generation and validation. Journal of Chemical Physics 134, 1-23.

46. Prinz, J.-H., Keller, B., and Noé, F. (2011). Probing molecular kinetics with Markov models: metastable states, transition pathways and spectroscopic observables. Physical Chemistry Chemical Physics 13, 16912.

47. Schwantes, C. R., and Pande, V. S. (2013). Improvements in Markov State Model construction reveal many non-native interactions in the folding of NTL9. Journal of Chemical Theory and Computation 9, 20002009.

48. Pérez-Hernández, G., Paul, F., Giorgino, T., De Fabritiis, G., and Noé, F. (2013). Identification of slow molecular order parameters for Markov model construction. Journal of Chemical Physics 139, 15102.
Izrailev, S., Stepaniants, S., Isralewitz, B., Kosztin, D., Lu, H., Molnar, F., Wriggers, W., and Schulten, K. In Computational Molecular Dynamics: Challenges, Methods, Ideas, Deuflhard, P., Hermans, J., Leimkuhler, B., Mark, A. E., Reich, S., and Skeel, R. D., Eds.; Springer-Verlag: Berlin, 1998, pp 39-65.

50. Dudko, O. K., Hummer, G., and Szabo, A. (2008). Theory, analysis, and interpretation of single-molecule force spectroscopy experiments. Proceedings of the National Academy of Sciences 105, 15755-15760.

51. Rico, F., Russek, A., González, L., Grubmüller, H., and Scheuring, S. (2019). Heterogeneous and ratedependent streptavidin-biotin unbinding revealed by high-speed force spectroscopy and atomistic simulations. Proceedings of the National Academy of Sciences 116, 6594-6601.

52. Cheng, F., Shen, J., Luo, X., Jiang, H., and Chen, K. (2002). Steered Molecular Dynamics Simulations on the "Tail Helix Latch" Hypothesis in the Gelsolin Activation Process. Biophysical Journal 83, 753-762.

53. Guzmán, D. L., Roland, J. T., Keer, H., Kong, Y. P., Ritz, T., Yee, A., and Guan, Z. (2008). Using steered molecular dynamics simulations and single-molecule force spectroscopy to guide the rational design of biomimetic modular polymeric materials. Polymer 49, 3892-3901.

54. Nielbo, S., Thomsen, J. K., Graversen, J. H., Jensen, P. H., Etzerodt, M., Poulsen, F. M., and Thøgersen, H. C. (2004). Structure of the plasminogen kringle 4 binding calcium-free form of the C-type lectin-like domain of tetranectin. Biochemistry 43, 8636-8643.

55. Poget, S. F., Freund, S. M. V. V., Howard, M. J., and Bycroft, M. (2001). The ligand-binding loops in the tunicate C-type lectin TC14 are rigid. Biochemistry 40, 10966-10972.

56. Ng, K. K. S., Park-Snyder, S., and Weis, W. I. (1998). $\mathrm{Ca}^{2+}$-dependent structural changes in C-type mannosebinding proteins. Biochemistry 37, 17965-17976.

57. Kim, M. O., Blachly, P. G., and McCammon, J. A. (2015). Conformational Dynamics and Binding Free Energies of Inhibitors of BACE-1: From the Perspective of Protonation Equilibria. PLOS Computational Biology 11, 1-28.

58. Pace, C. N., Grimsley, G. R., and Scholtz, J. M. (2009). Protein Ionizable Groups: $\mathrm{p} K$ Values and Their Contribution to Protein Stability and Solubility. Journal of Biological Chemistry 284, 13285-13289. 
59. Søndergaard, C. R., Olsson, M. H. M., Rostkowski, M., and Jensen, J. H. (2011). Improved Treatment of Ligands and Coupling Effects in Empirical Calculation and Rationalization of $\mathrm{p} K_{\mathrm{a}}$ Values. Journal of Chemical Theory and Computation 7, 2284-2295.

60. Olsson, M. H. M., Søndergaard, C. R., Rostkowski, M., and Jensen, J. H. (2011). PROPKA3: Consistent Treatment of Internal and Surface Residues in Empirical $\mathrm{p} K_{\mathrm{a}}$ Predictions. Journal of Chemical Theory and Computation 7, 525-537.

61. Khandogin, J., and Brooks, C. L. (2005). Constant $\mathrm{pH}$ Molecular Dynamics with Proton Tautomerism. Biophysical Journal 89, 141-157.

62. Lee, J., Miller, B. T., Damjanović, A., and Brooks, B. R. (2015). Enhancing Constant-pH Simulation in Explicit Solvent with a Two-Dimensional Replica Exchange Method. Journal of Chemical Theory and Computation 11, PMID: 26575555, 2560-2574.

63. Radak, B. K., Chipot, C., Suh, D., Jo, S., Jiang, W., Phillips, J. C., Schulten, K., and Roux, B. (2017). Constant-pH Molecular Dynamics Simulations for Large Biomolecular Systems. Journal of Chemical Theory and Computation 13, PMID: 29111720, 59335944.

64. Paasche, A., Schirmeister, T., and Engels, B. (2013). Benchmark Study for the Cysteine-Histidine Proton Transfer Reaction in a Protein Environment: Gas Phase, COSMO, QM/MM Approaches. Journal of Chemical Theory and Computation 9, PMID: 26587634, 17651777.

65. Duster, A. W., and Lin, H. (2019). Tracking Proton Transfer through Titratable Amino Acid Side Chains in Adaptive QM/MM Simulations. Journal of Chemical Theory and Computation 15, PMID: 31553601, 57945809.

66. Jonker, C. T. H., Deo, C., Zager, P. J., Tkachuk, A. N., Weinstein, A. M., Rodriguez-Boulan, E., Lavis, L. D., and Schreiner, R. (2020). Accurate measurement of fast endocytic recycling kinetics in real time. Journal of Cell Science 133, jcs231225.

67. J., Y., Wilson, J., and Aksimentiev, A. (2016). Improved model of hydrated calcium ion for molecular dynamics simulations using classical biomolecular force fields. Biopolymers 105, 752-763.

68. Timr, S., Kadlec, J., Srb, P., Ollila, O. H. S., and Jungwirth, P. (2018). Calcium Sensing by Recoverin: Effect of Protein Conformation on Ion Affinity. Journal of Physical Chemistry Letters 9, 1613-1619.
69. Saxena, A., and Sept, D. (2013). Multisite Ion Models That Improve Coordination and Free Energy Calculations in Molecular Dynamics Simulations. Journal of Chemical Theory and Computation 9, 3538-3542.

70. Jing, Z., Liu, C., Cheng, S. Y., Qi, R., Walker, B. D., Piquemal, J.-P., and Ren, P. (2019). Polarizable Force Fields for Biomolecular Simulations: Recent Advances and Applications. Annual Review of Biophysics 48, 371-394.

71. Berendsen, H. J. C., van der Spoel, D., and van Drunen, R. (1995). GROMACS: A message-passing parallel molecular dynamics implementation. Computer Physics Communications 91, 43-56.

72. Lindahl, E., Hess, B., and van der Spoel, D. (2001). GROMACS 3.0: A package for molecular simulation and trajectory analysis. Journal of Molecular Modeling 7, 306-317.

73. van der Spoel, D., Lindahl, E., Hess, B., Groenhof, G., Mark, A. E., and Berendsen, H. J. (2005). GROMACS: Fast, flexible, and free. Journal of Computational Chemistry 26, 1701-1718.

74. Hess, B., Kutzner, C., van der Spoel, D., and Lindahl, E. (2008). GROMACS 4: Algorithms for Highly Efficient, Load-Balanced, and Scalable Molecular Simulation. Journal of Chemical Theory and Computation 4, 435447.

75. Pronk, S., Páll, S., Schulz, R., Larsson, P., Bjelkmar, P., Apostolov, R., Shirts, M. R., Smith, J. C., Kasson, P. M., van der Spoel, D., Hess, B., and Lindahl, E. (2013). GROMACS 4.5: A high-throughput and highly parallel open source molecular simulation toolkit. Bioinformatics 29, 845-854.

76. Páll, S., Abraham, M. J., Kutzner, C., Hess, B., and Lindahl, E., Tackling exascale software challenges in molecular dynamics simulations with GROMACS; Markidis, S., and Laure, E., Eds.; Springer: 2015; Vol. 8759.

77. Abraham, M. J., Murtola, T., Schulz, R., Páll, S., Smith, J. C., Hess, B., and Lindahl, E. (2015). GROMACS: High performance molecular simulations through multi-level parallelism from laptops to supercomputers. SoftwareX 1-2, 19-25.

78. Lindorff-Larsen, K., Piana, S., Palmo, K., Maragakis, P., Klepeis, J. L., Dror, R. O., and Shaw, D. E. (2010). Improved side-chain torsion potentials for the Amber ff99SB protein force field. Proteins: Structure, Function, and Bioinformatics 78, 1950-1958. 
bioRxiv preprint doi: https://doi.org/10.1101/2020.03.11.986851; this version posted February 15,2021 . The copyright holder for this preprint (which was not certified by peer review) is the author/funder, who has granted bioRxiv a license to display the preprint in perpetuity. It is made available under aCC-BY-NC 4.0 International license.

79. Jorgensen, W. L., Chandrasekhar, J., Madura, J. D., Impey, R. W., and Klein, M. L. (1983). Comparison of

simple potential functions for simulating liquid water.

The Journal of Chemical Physics 79, 926-935. 